\title{
Tai Chi Chuan for the Primary Prevention of Stroke in Middle-Aged and Elderly Adults: A Systematic Review
}

\author{
Guohua Zheng, ${ }^{1}$ Maomao Huang, ${ }^{1}$ Feiwen Liu, ${ }^{1}$ Shuzhen Li, ${ }^{1}$ Jing Tao, ${ }^{1}$ and Lidian Chen ${ }^{2}$ \\ ${ }^{1}$ College of Rehabilitation Medicine, Fujian University of Traditional Chinese Medicine, No. 1 Huatuo Road, \\ Shangjie University Town, Fuzhou 350108, China \\ ${ }^{2}$ Fujian University of Traditional Chinese Medicine, No. 1 Huatuo Road, Shangjie University Town, Fuzhou 350108, China
}

Correspondence should be addressed to Lidian Chen; lidianchen87@163.com

Received 4 November 2014; Accepted 4 January 2015

Academic Editor: I-Min Liu

Copyright (c) 2015 Guohua Zheng et al. This is an open access article distributed under the Creative Commons Attribution License, which permits unrestricted use, distribution, and reproduction in any medium, provided the original work is properly cited.

\begin{abstract}
Background. Stroke is a major healthcare problem with serious long-term disability and is one of the leading causes of death in the world. Prevention of stroke is considered an important strategy. Methods. Seven electronic databases were searched. Results. 36 eligible studies with a total of 2393 participants were identified. Primary outcome measures, TCC exercise combined with other intervention had a significant effect on decreasing the incidence of nonfatal stroke $(n=185, \mathrm{RR}=0.11,95 \% \mathrm{CI} 0.01$ to $0.85, P=0.03)$ and $\operatorname{CCD}(n=125, \mathrm{RR}=0.33,95 \% \mathrm{CI} 0.11$ to $0.96, P=0.04)$. For the risk factors of stroke, pooled analysis demonstrated that TCC exercise was associated with lower body weight, BMI, FBG level, and decreasing SBP, DBP, plasma TC, and LDL-C level regardless of the intervention period less than half a year or more than one year and significantly raised HDL-C level in comparison to nonintervention. Compared with other treatments, TCC intervention on the basis of the same other treatments in patients with chronic disease also showed the beneficial effect on lowering blood pressure. Conclusion. The present systematic review indicates that TCC exercise is beneficially associated with the primary prevention of stroke in middle-aged and elderly adults by inversing the high risk factors of stroke.
\end{abstract}

\section{Introduction}

Although the rates of stroke mortality have declined over recent decades in most of developed countries, stroke still occupies the third commonest cause of mortality following heart disease and cancer, and resulting in around 6 million deaths annually in the world [1]. In China, approximately 2 million adults suffer a new stoke every year [2], and 6 million adults are currently standing stroke [3]. It is estimated that 700,000 people sustain a new stroke each year in the United States and on average every 40 second someone in this country has a stroke [4]. According to WHO, stroke also was one of the leading causes of adult acquiring disability and a major contributor to health-care cost worldwide. Lifetime costs per patient are estimated at between US $\$ 59,800$ and US $\$ 230,000$ [5]. In the UK, the direct and indirect societal costs caused by stroke are about 8.9 billion pounds a year [6]. The risk of suffering from stroke is governed by more than 100 risk factors which are classified as nonmodifiable, potentially modifiable, and modifiable factors. Prevention for stroke, in which its strategy aims are to control the risk of stoke by modifying one or more modifiable risk factors, such as physical activity level, obesity, cholesterol levels, blood pressure, smoking status, and glucose intolerance, plays a crucial role in counteracting morbidity and mortality related to stroke and is considered to be the best approach in reducing the burden of stroke [7]. It has been estimated that 50\% of stroke are preventable through control of modifiable risk factors, in which exercise contributes an important part [8]. Regular exercise has favorable effects on controlling risk factors of stroke and reducing the incidence rate of a first-ever stroke $[9,10]$.

Tai Chi Chuan (TCC) exercise originated in China as a martial art is gentle and vigorous exercise with low impact and low-moderate intensity which involves a series of slow, continuous, and graceful body movements [11]. To date, TCC 
has developed into several styles which can be differentiated by the varying forms or postures, the order of the movement sequence, pace of movement, and the angle of knee flexion during the practice $[12,13]$. The commonly practiced styles include "Yang," "Chen," "Wu," or "Sun" styles among which the Yang style is the most popular and the Chen style is the oldest [12]. Though there are differences of posture and the position of the center of gravity, all styles incorporate slowness, rhythmic movements, relaxation, mental concentration, movement coordination, and flow into the next one with elements of meditation, body awareness, and imagery while breathing deeply [13].

TCC is a suitable exercise for people with different ages, different physical and health conditions, because it is easily accessible and of low cost, and can be easily implemented in the community setting. As an exercise for promoting health, TCC has been practiced for hundreds of years in China and is gradually acceptable in the West countries. A systematic review indicated that intensive TCC exercise shown some favorable effects on improving general cardiorespiratory fitness and its functional status, and was potentially beneficial for cardiovascular disease of elderly population [14]. A substantial amount of studies reported that TCC was efficient to control many risk factors of stroke [15-20], but there has not been a comprehensive systematic review to examine the primary preventive effect of TCC for stroke. The objective of the current study was to attempt to conduct a systematic review and meta-analysis of the existing studies on TCC exercise as an intervention for the primary prevention of stroke in middle-aged and elderly adults to draw more useful conclusions about the safety and efficacy of TCC in preventing stroke, and to offer recommendations for future research.

\section{Methods}

2.1. Literature Search. We searched the following electronic databases: PubMed Database, EMBASE (OVID) Database, Science Citation Index (SCI), Wanfang degree and conference papers database, China National Knowledge Infrastructure (CNKI), and Chinese Science and Technology Periodical Database (VIP) from their inception to 31 October 2013, and the Cochrane Central Register of Controlled Trials (Cochrane Library, 2013, Issue 3). The search terms included "Stroke," "Tai Ji," "cerebral hemorrhage," "infarction," "blood pressure," "cholesterol," and "blood sugar." The details of search strategy are listed in Appendix A. In addition, we checked references list of reviews and retrieved articles for additional studies. No language restrictions were applied to any searches.

2.2. Eligibility Criteria. Randomized controlled trials (RCTs), quasirandomized controlled trials (quasi-RCTs), prospective nonrandomized controlled trials (NRCTs), self-controlled trials and first stage of cross-over trials whether published or unpublished were included. The target population was aged 30 or older with or without high risk factors of stroke. Type of TCC exercise was not limited at a frequency of at least 30 minutes per time and 3 times per week for 4 weeks. Trials with comparison of TCC exercise versus nonintervention or TCC exercise plus other treatment versus same other treatment were included, but trials with comparison of TCC exercise versus other exercise intervention or TCC exercise plus other treatment versus other exercise intervention plus same other treatment were excluded. Primary outcome measures were incidence rates of fatal or nonfatal stroke or cardiacerebrovascular disease (CCD), and secondary outcome measures included any modification risk factor of stroke, such as blood pressure, blood lipids, fasting blood glucose (FBG).

2.3. Study Identification and Data Extraction. Two reviewers (HMM and LSZ) assessed the eligibility of the searched studies independently. The full-text articles that met the eligible criteria were obtained, and the relevant references were retrieved according to predefined eligibility criteria. Data concerning details of participants' characteristics, study methods, interventions, and outcomes were extracted independently by two reviewers (HMM and LSZ) through using a form based on predefined selection criteria. We resolved any disagreements of study identification and data extraction by consensus and consulted a third reviewer $(\mathrm{ZGH})$ if disagreements persisted. We also contacted original author to provide additional relevant information if necessary.

2.4. Risk of Bias in Individual Studies. Risk of bias of the included studies was assessed using the Cochrane Collaboration's tool for assessing risk of bias by two reviewers (HMM, LFW) independently [21]. Six following criteria were applied: adequate sequence generation, concealment of allocation, blinded of primary outcomes, adequately addressed incomplete outcome data, free from selective reporting, and free of other risk of bias [21]. In addition, we assessed the baseline characteristics between the comparison groups. The disagreements between two reviewers were resolved through discussion.

2.5. Statistical Analysis. Data were processed in accordance with the Cochrane Handbook for Systematic Reviews of Interventions [20]. Meta-analysis was carried out using Review Manager Software 5.2 (2011, Cochrane Collaboration and Updated Software). Relative risk (RR) with 95\% confidence interval (CI) was calculated for dichotomous variables. For continuous outcomes, net changes were compared a mean difference (MD) or standardized mean difference (SMD), and corresponding 95\% confidence interval (CI) were calculated for each study. Heterogeneity test of each outcome was conducted using the Chi-square test with non significance $(P>0.05)$ indicating no heterogeneity among studies [22]. Degree of heterogeneity was evaluated using $I^{2}$ statistic [22]. Where there was no heterogeneity, a fixedeffect model was performed in meta-analysis, otherwise random effects model was used. If substantial heterogeneity was detected, the review authors looked for possible explanations, and considered to use the following options: provide a narrative overview, not aggregate the studies at 


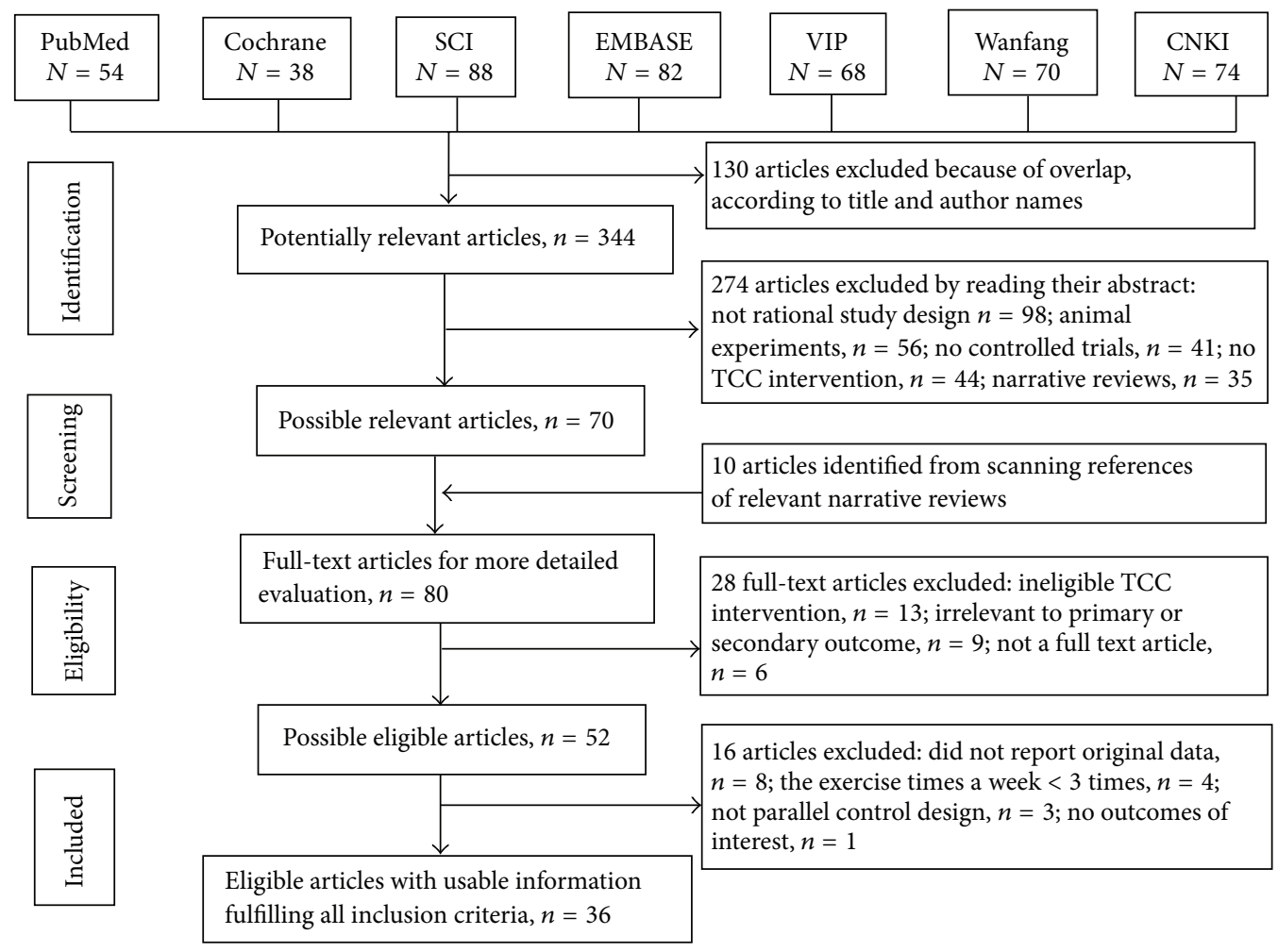

FIgURE 1: Flow diagram for search and selection of the included studies.

all, or use a random-effect model with appropriate cautious interpretation. Subgroup analysis or sensitivity analysis was applied to explore the cause of heterogeneity among studies.

\section{Results}

3.1. Study Identification. Figure 1 summarizes the flow of the literature search and selection process. A total of 474 records were identified from the relevant databases, and 130 duplicate records were excluded. Among the 344 potential articles, 274 were excluded by reading the title and abstract. 80 full-text articles including ten of which was identified from the reference lists were evaluated for their eligibility. 44 articles were further excluded because they did not meet the inclusion criteria. A list of excluded studies can be found in Appendix B. Finally, 36 studies with a total of 2393 participants were eligible to be included in this systematic review [23-58].

3.2. Characteristics of Included Studies. Characteristics of the methods, participants, intervention, comparison group and outcome measures of each included studies in this review were shown in Table 1 . Among the 36 included studies, only one [36] was conducted in Mexico and others conducted in China. 2 of 36 articles were reported in English, and the remaining reported in Chinese. Participants in the included studies involved in healthy individuals or patients with chronic diseases, and their ages ranged from 30 to 82 years. 22 trials [23-44] compared TCC with nonintervention. The remaining trials were designed comparing TCC plus conventional treatment with same conventional treatment. In these trials, frequency of TCC intervention was at least 3 times one week, more than 30 minutes per time with at least 1 month duration. Two studies reported the primary outcomes including the incidence of fatal, nonfatal stroke and cardia-cerebrovascular disease $[48,58]$, and others mainly focused on the risk factors of stroke such as body weight, blood pressure, blood lipids, or blood glucose. No studies reported the adverse events.

3.3. Methodological Quality of Included Studies. Details of the risk of bias assessed for each of the included studies are summarized in Figure 2 and Appendix C. Of the 36 included studies, 23 studies reported randomization allocation, but only 4 studies [27, 29, 48, 52] described the method of randomization by using random number tables or stratified random distribution. No study reported the allocation concealment. Only one trial [37] clearly described the outcome assessors blinded. Five studies [28, 31, 33, 36, 58] reported numbers of participants who dropped out, and one of five [33] performed the intention to treat analysis and another [58] reported follow-up period. As a whole, 8 out of 36 studies $(22.2 \%)$ were judged as at high risk of bias because one or more main aspects of the bias assessment was labeled high, and other studies were judged as at unclear risk of bias. We 


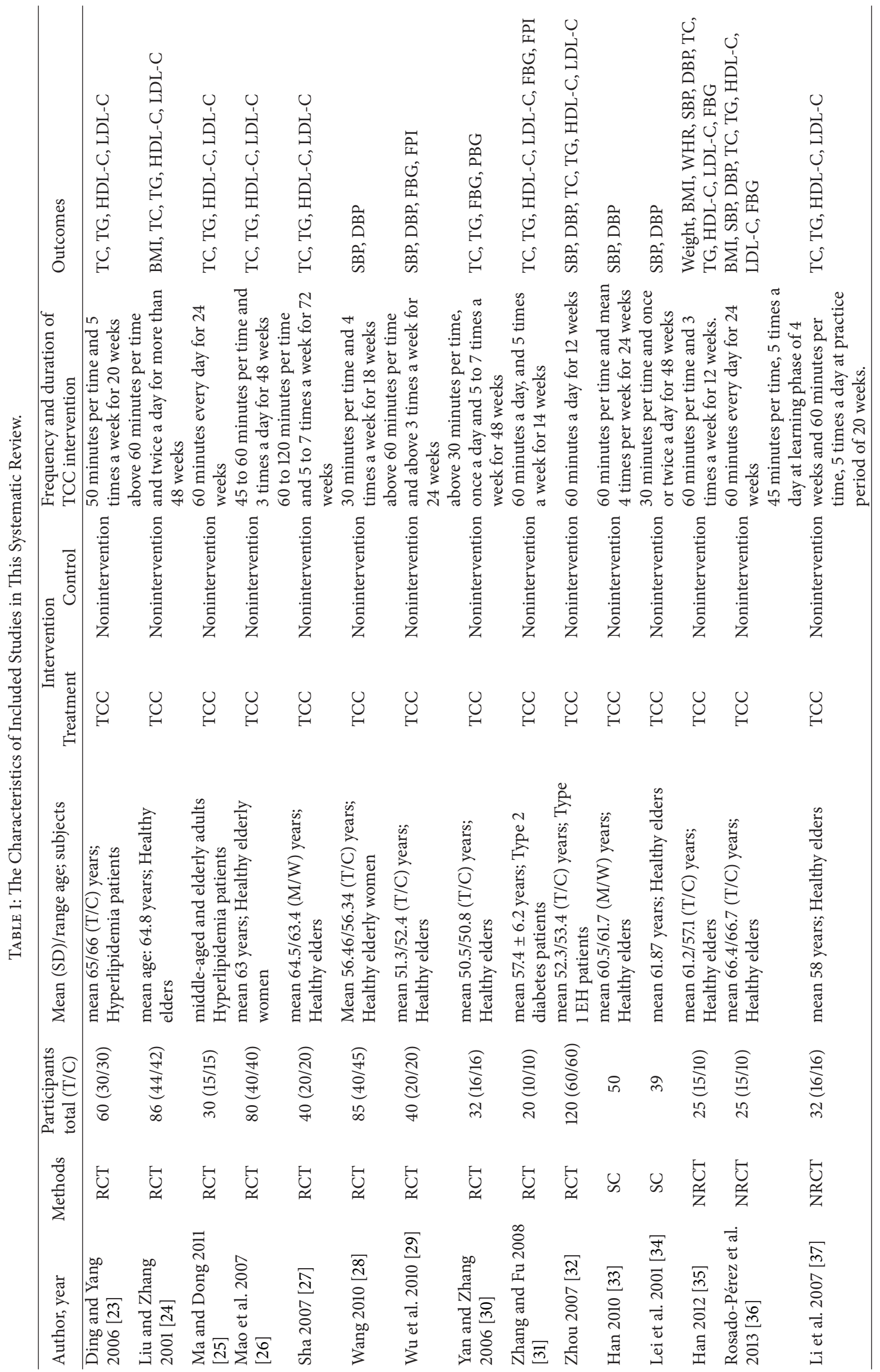




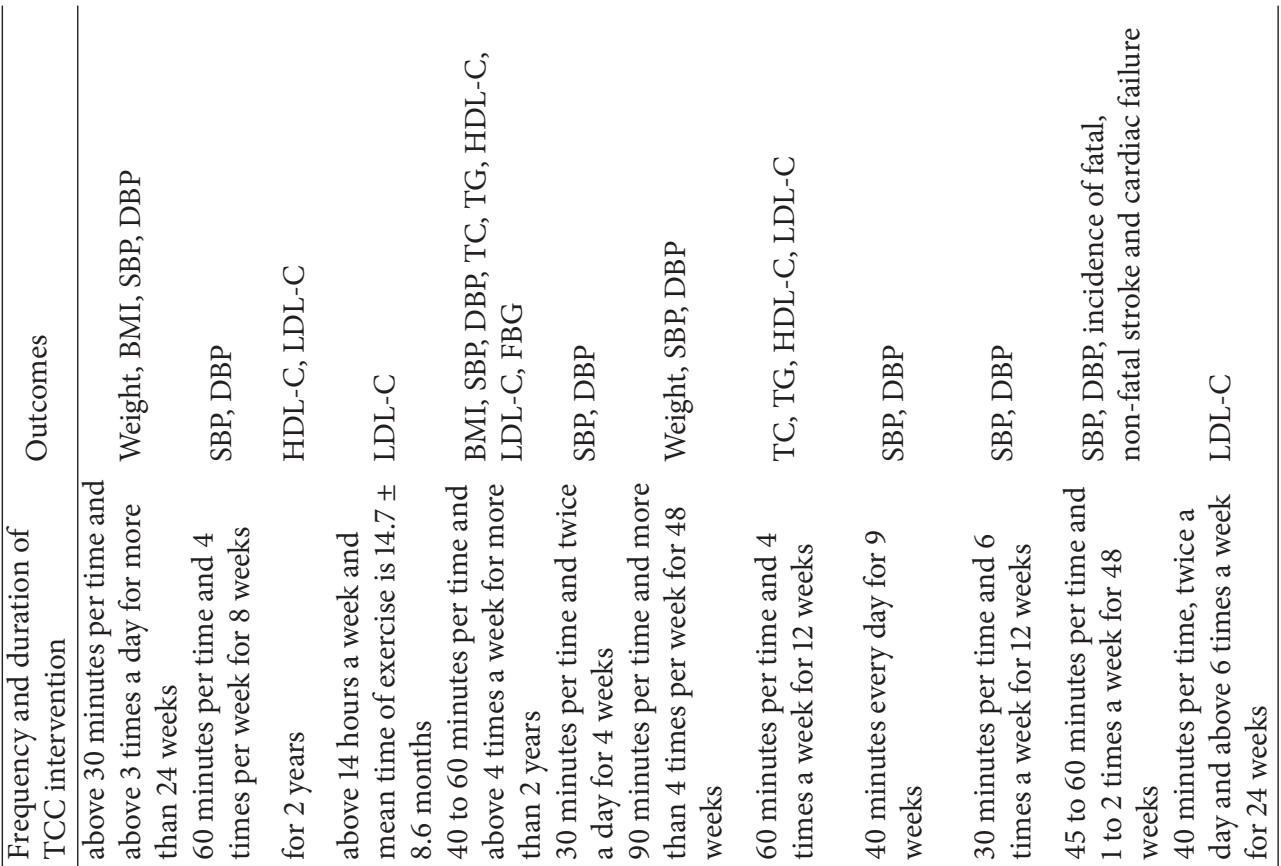




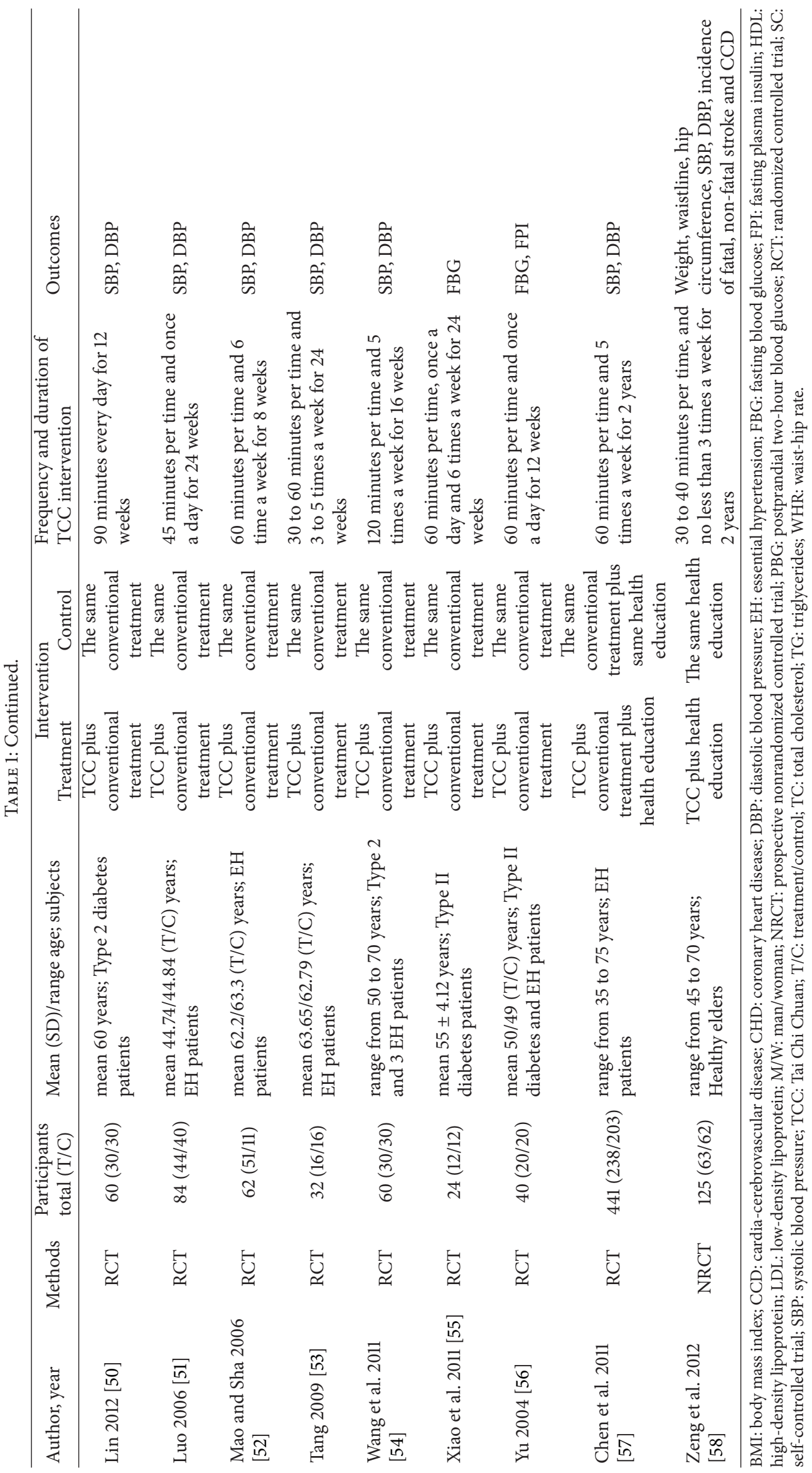




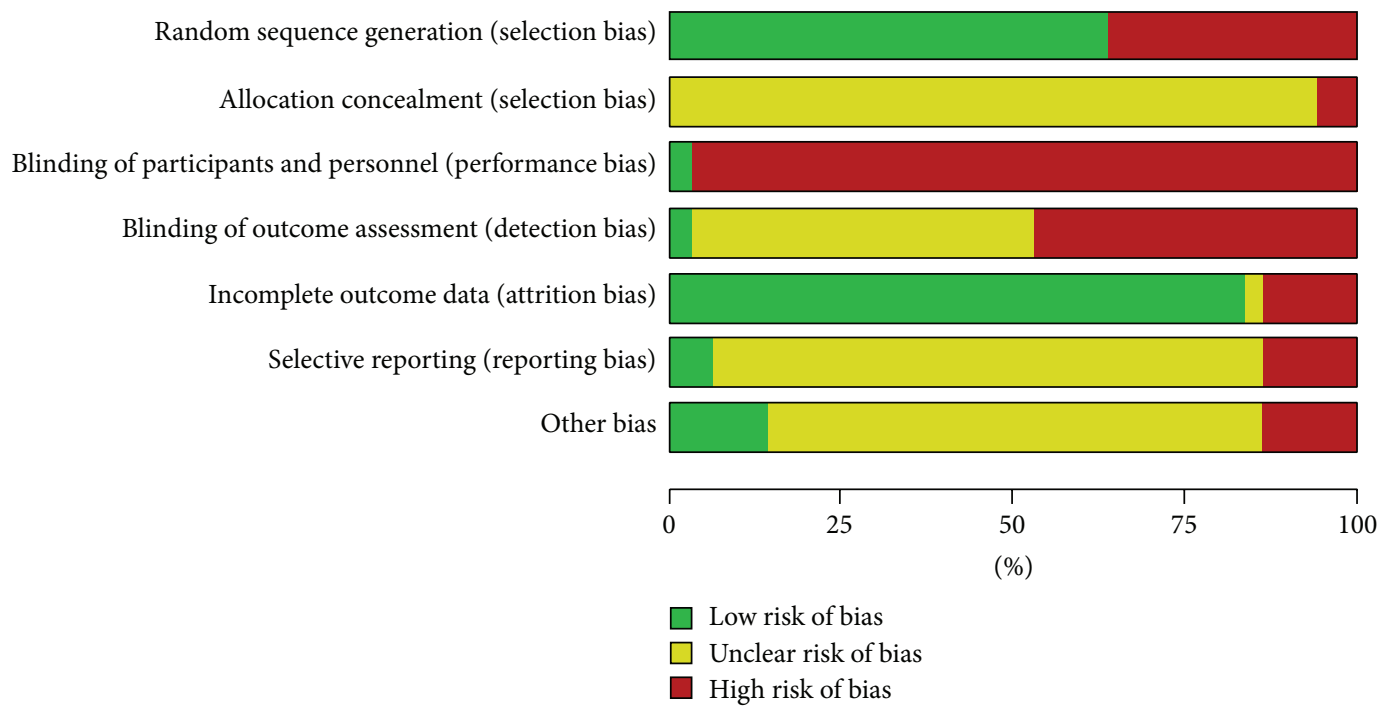

FIGURE 2: "Risk of bias" graph: review authors' judgments about each risk of bias item presented as percentages across all included studies.

TABLE 2: The effect of TCC on incidence of fatal stroke, non-fatal stroke, cardia-cerebrovascular disease, and cardiac failure.

\begin{tabular}{|c|c|c|c|c|c|c|}
\hline Outcome or subgroup title & $\begin{array}{l}\text { Number } \\
\text { of studies }\end{array}$ & $\begin{array}{l}\text { Number of } \\
\text { participants }\end{array}$ & $\begin{array}{l}\text { Effect size; risk ratio (M-H, } \\
\text { fixed, } 95 \% \mathrm{CI})\end{array}$ & $P$ value & $I^{2}$ & $\mathrm{P}_{\text {Heterogeneity }}$ \\
\hline \multicolumn{7}{|c|}{ Tai Chi Chuan plus other intervention versus same other intervention } \\
\hline Incidence of nonfatal stroke & 2 & 185 & $0.11[0.01,0.85]$ & 0.03 & $0 \%$ & 0.65 \\
\hline Incidence of fatal stroke & 2 & 185 & $0.33[0.05,2.05]$ & 0.23 & $0 \%$ & 0.64 \\
\hline Incidence of CCD & 1 & 125 & $0.33[0.11,0.96]$ & 0.04 & & \\
\hline Incidence of cardiac failure & 1 & 60 & $0.17[0.02,1.30]$ & 0.09 & & \\
\hline
\end{tabular}

CCD: cardia-cerebrovascular disease.

tried to contact the original authors but most of them had no response.

\subsection{Measures of Effect}

3.4.1. Incidence of Fatal, Nonfatal Stroke, Cardia-Cerebrovascular Disease and Cardiac Failure. Two studies with 185 elders reported the primary outcomes including incidence of fatal and nonfatal stroke $[48,58]$, the incidence of CCD [45], and the incidence of cardiac failure [58]. Compared to other intervention, TCC exercise combined with other intervention had a significant effect to decrease the incidence of nonfatal stroke $(n=185, \mathrm{RR}=0.11,95 \% \mathrm{CI} 0.01$ to $0.85, P=0.03)$ and CCD $(n=125, \mathrm{RR}=0.33,95 \%$ CI 0.11 to $0.96, P=0.04)$, but the significant effects were not observed on the incidence of fatal stroke $(n=185, \mathrm{RR}=0.33,95 \% \mathrm{CI} 0.05$ to $2.05, P=0.23)$ and cardiac failure $(n=60, \mathrm{RR}=0.17,95 \%$ CI 0.02 to 1.30 , $P=0.09$ ) (Table 2).

3.4.2. Body Weight, BMI, WHR, Waistline, Hip Circumference. Meta-analysis showed the significant differences between TCC intervention and nonintervention on decreasing body weight $(n=241, \mathrm{MD}=-3.21 \mathrm{~kg}, 95 \% \mathrm{CI}-5.18$ to -1.24 , $\left.P=0.001, I^{2}=0 \%\right)$, and body mass index (BMI) $(n=381$,
$\mathrm{MD}=-1.01 \mathrm{~kg} / \mathrm{m}^{2}, 95 \% \mathrm{CI}-1.34$ to $-0.69, P<0.00001, I^{2}=$ $3 \%)$ in healthy participants. One study [35] with 25 healthy participants also showed TCC intervention was efficient to reduce waist-hip ratio (WHR) in comparison to nonintervention $(\mathrm{MD}=-2.40,95 \% \mathrm{CI}-4.53$ to $-0.27, P=0.03)$ (Table 3$)$.

Another study [58] in 125 healthy participants measured body weight, waistline, and hip circumference after two years intervention, and results showed that TCC exercise plus health education were better than health education alone on reducing body weight $(\mathrm{MD}=-4.30 \mathrm{~kg}, 95 \% \mathrm{CI}-7.29$ to -1.31 , $P=0.005)$, waistline ( $\mathrm{MD}=-7.00 \mathrm{~cm}, 95 \% \mathrm{CI}-10.1$ to -3.90 , $P<0.00001)$, and hip circumference $(\mathrm{MD}=-4.60 \mathrm{~cm}, 95 \%$ CI -6.91 to $-2.29, P<0.00001$ ) (Table 3$)$.

3.4.3. Blood Pressure. 12 studies with 832 healthy participants comparing TCC intervention with nonintervention and 10 studies mainly involving in patients with chronic diseases comparing TCC intervention plus other intervention with same other intervention (conventional treatment or health education) reported the effect of TCC intervention for blood pressure. Compared to nonintervention, TCC intervention with intervention period for more than one year had significant reduction on systolic blood pressure (SBP) $(n=198$, $\mathrm{MD}=-9.58 \mathrm{mmHg}$, $95 \% \mathrm{CI}-14.54$ to $-4.61, P=0.0002$, 
TABLE 3: The effect of TCC on body weight, body mass index, waist-hip ratio, waistline, and hip circumference.

\begin{tabular}{|c|c|c|c|c|c|c|}
\hline Outcome or subgroup title & $\begin{array}{c}\text { Number } \\
\text { of } \\
\text { studies }\end{array}$ & $\begin{array}{l}\text { Number of } \\
\text { participants }\end{array}$ & $\begin{array}{l}\text { Effect size; mean difference } \\
\quad(\mathrm{IV} \text {, fixed, 95\% CI) }\end{array}$ & $P$ value & $I^{2}$ & $\mathrm{P}_{\text {Heterogeneity }}$ \\
\hline \multicolumn{7}{|c|}{ Tai Chi Chuan versus nonintervention } \\
\hline Body weight (kg) & 3 & 241 & $-3.21[-5.18,-1.24]$ & 0.001 & $0 \%$ & 0.58 \\
\hline BMI $\left(\mathrm{kg} / \mathrm{m}^{2}\right)$ & 5 & 381 & $-1.01[-1.34,-0.69]$ & $<0.00001$ & $3 \%$ & 0.39 \\
\hline WHR & 1 & 25 & $-2.40[-4.53,-0.27]$ & 0.03 & & \\
\hline \multicolumn{7}{|c|}{ Tai Chi Chuan plus health education versus same health education } \\
\hline Body weight (kg) & 1 & 125 & $-4.30[-7.29,-1.31]$ & 0.005 & & \\
\hline Waistline $(\mathrm{cm})$ & 1 & 125 & $-7.00[-10.10,-3.90]$ & $<0.00001$ & & \\
\hline Hip circumference $(\mathrm{cm})$ & 1 & 125 & $-4.60[-6.91,-2.29]$ & $<0.00001$ & & \\
\hline
\end{tabular}

BMI: body mass index; WHR: waist-hip ratio.

TABLE 4: The effect of TCC on blood pressure.

\begin{tabular}{|c|c|c|c|c|c|c|}
\hline Outcome or subgroup title & $\begin{array}{c}\text { Number } \\
\text { of } \\
\text { studies }\end{array}$ & $\begin{array}{l}\text { Number of } \\
\text { participants }\end{array}$ & $\begin{array}{l}\text { Effect size; std. mean difference (IV, } \\
\text { random, 95\% CI) }\end{array}$ & $P$ value & $I^{2}$ & $\mathrm{P}_{\text {Heterogeneity }}$ \\
\hline \multicolumn{7}{|c|}{ Tai Chi Chuan versus nonintervention for blood pressure } \\
\hline \multicolumn{7}{|l|}{ SBP (mmHg) } \\
\hline Intervention for less than half a year & 9 & 634 & $-11.98[-17.50,-6.47]$ & $<0.0001$ & $90 \%$ & $<0.00001$ \\
\hline Intervention for more than 1 year & 3 & 198 & $-9.58[-14.54,-4.61]$ & 0.0002 & $0 \%$ & 0.45 \\
\hline \multicolumn{7}{|l|}{$\mathrm{DBP}(\mathrm{mmHg})$} \\
\hline Intervention for less than half a year & 9 & 634 & $-6.11[-9.92,-2.29]$ & 0.002 & $90 \%$ & $<0.00001$ \\
\hline Intervention for more than 1 year & 3 & 198 & $-4.00[-7.44,-0.56]$ & 0.02 & $11 \%$ & 0.32 \\
\hline \multicolumn{7}{|c|}{ Tai Chi Chuan plus other interventions versus same other interventions } \\
\hline \multicolumn{7}{|l|}{ SBP $(\mathrm{mmHg})$} \\
\hline Intervention for less than half a year & 7 & 406 & $-14.21[-17.54,-10.88]$ & $<0.00001$ & $43 \%$ & 0.11 \\
\hline Intervention for more than 1 year & 3 & 624 & $-8.29[-9.63,-6.95]$ & $<0.00001$ & $0 \%$ & 0.92 \\
\hline \multicolumn{7}{|l|}{$\mathrm{DBP}(\mathrm{mmHg})$} \\
\hline Intervention for less than half an year & 7 & 406 & $-7.08[-9.06,-5.09]$ & $<0.00001$ & $47 \%$ & 0.08 \\
\hline Intervention for more than 1 year & 3 & 624 & $-4.56[-6.45,-2.67]$ & 0.0002 & $38 \%$ & 0.20 \\
\hline
\end{tabular}

SBP: systolic blood pressure; DBP: diastolic blood pressure.

$\left.I^{2}=0 \%\right)$, or diastolic blood pressure (DBP) $(n=198, \mathrm{MD}=$ $-4.00 \mathrm{mmHg}$, $95 \% \mathrm{CI}-7.44$ to $\left.-0.56, P=0.02, I^{2}=11 \%\right)$. The pooled results of TCC intervention for less than half a year also showed the significant difference between TCC intervention and nonintervention on SBP $(n=634, \mathrm{MD}=$ $-11.98 \mathrm{mmHg}, 95 \% \mathrm{CI}-17.50$ to $-6.47, P<0.0001)$ and DBP $(n=634, \mathrm{MD}=-6.11 \mathrm{mmHg}, 95 \% \mathrm{CI}-9.92$ to $-2.29, P=$ 0.002) (Table 4). But their heterogeneities among studies also were substantive (for both SBP and DBP $I^{2}=90 \%$ ), sensitivity analysis, for SBP, after excluding one study with a low quality score [38] and the self-controlled trial [33], the effect size remained statistical significance with acceptable heterogeneity $(n=378, \mathrm{MD}=-15.15 \mathrm{mmHg}, 95 \% \mathrm{CI}-19.60$ to $\left.-10.71, P<0.00001, I^{2}=68 \%\right)$; for DBP, after excluding one study [28], the effect size was reduced but remained statistical significance with moderate heterogeneity
( $n=547, \mathrm{MD}-4.06 \mathrm{mmHg}, 95 \% \mathrm{CI}-6.12$ to $-1.99, P=$ $\left.0.0001, I^{2}=63 \%\right)$.

Table 4 also showed that TCC intervention plus other intervention for patients with chronic diseases was far superior to same other intervention used alone regarding blood pressure reduction. There were significant differences between comparison groups after intervention regardless of less than half a year (SBP: $n=406, \mathrm{MD}=-14.21 \mathrm{mmHg}, 95 \% \mathrm{CI}$ -17.54 to $-10.88, P<0.00001$, random model; DBP: $n=406$, $\mathrm{MD}=-7.08 \mathrm{mmHg}$, 95\% CI -9.06 to $-5.09, P<0.00001$, random model), or more than one year (SBP: $n=624, \mathrm{MD}=$ $-8.29 \mathrm{mmHg}$, 95\% CI -9.63 to $-6.95, P<0.00001$, random model; DBP: $n=624, \mathrm{MD}=-4.56 \mathrm{mmHg}, 95 \% \mathrm{CI}-6.45$ to $-2.67, P<0.00001$, random model), and the heterogeneity among studies was also expressed a acceptable range from $I^{2}=0 \%$ to $I^{2}=60 \%$. 
3.4.4. Blood Lipid Levels (TC, TG, HDL-C, and LDL-C). There were 14 studies mainly involving in healthy participants comparing TCC intervention with nonintervention and 2 studies with 98 patients with chronic diseases comparing TCC intervention plus conventional treatment with same conventional treatment. They reported the effects of TCC for blood lipid levels including total cholesterol (TC), triglycerides (TG), high-density lipoprotein cholesterol (HDL-C) or lowdensity lipoprotein cholesterol (LDL-C).

For TC, TCC intervention showed a reduction of TC level at intervention period less than half a year $(n=340, \mathrm{SMD}=$ $-0.87,95 \%$ CI -1.66 to $-0.08, P=0.03, I^{2}=90 \%$ ) or more than one year $(n=298, \mathrm{SMD}=-0.32,95 \% \mathrm{CI}-0.63$ to $\left.-0.02, P=0.04, I^{2}=40 \%\right)$ in comparison to nonintervention (Table 5). Since there was significant heterogeneity in the comparison of subgroup with less than half a year intervention period $\left(I^{2}=90 \%\right)$, we examined the data carefully and found one study [23] deviated from the others. We performed a sensitive analysis through removing this study, and got a similar result with no heterogeneity $(n=280$, SMD $-0.43,95 \%$ CI -0.66 to $\left.-0.19, P=0.0005, I^{2}=0 \%\right)$. There was one study [45] with 60 patients with chronic disease reported TCC intervention plus conventional treatment versus same conventional treatment, and result showed a significant reduction of TC level $(\mathrm{MD}=-1.76 \mathrm{mmol} / \mathrm{L}, 95 \% \mathrm{CI}$ -2.02 to $-1.50, P<0.00001$ ) (Table 5).

Subgroup analysis according to different TCC intervention period found significant reduction of TG level at TCC intervention period for less than half a year $(n=340$, SMD $=-0.58,95 \% \mathrm{CI}-1.03$ to $\left.-0.14, P=0.01, I^{2}=72 \%\right)$, or more than one year $(n=298$, SMD $=-0.67,95 \%$ CI -0.90 to $-0.43, P<0.00001, I^{2}=0 \%$ ) (Table 5). One study [45] comparing TCC intervention plus conventional treatment with same conventional treatment reported there was no significant difference on serum TG level in patients with chronic disease $(n=60, \mathrm{MD}=-0.05 \mathrm{mmol} / \mathrm{L}, 95 \% \mathrm{CI}$ -0.27 to $0.17, P=0.66$ ) (Table 5).

Compared to nonintervention, TCC intervention showed a increase of HDL-C level regardless of intervention period for less than half a year $(n=340, \mathrm{SMD}=0.77,95 \%$ CI 0.01 to $\left.1.53, P=0.05, I^{2}=90 \%\right)$ or more than one year $(n=335$, $\mathrm{SMD}=0.88,95 \%$ CI 0.44 to $1.32, P<0.0001, I^{2}=73 \%$ ) (Table 5). But the heterogeneity in subgroup analysis of TCC intervention period more than one year were substantive with $I^{2}$ value being $90 \%$, and the subtotal meat-analysis showed no significant difference $(n=280, \mathrm{SMD}=0.40,95 \% \mathrm{CI}-0.07$ to $0.86, P=0.09, I^{2}=67 \%$ ) after sensitivity analysis was performed through excluded one study [23]. One study [45] with 60 patients with chronic disease reported TCC intervention plus conventional treatment versus same conventional treatment on HDL-C level, and result showed a significant increase of HDL-C level $(\mathrm{MD}=0.20 \mathrm{mmol} / \mathrm{L}, 95 \% \mathrm{CI}$ 0.16 to $0.24, P<0.00001$ ) (Table 5 ).

Subgroup analysis showed TCC intervention in comparison to nonintervention could significantly reduce the LDL-C level at intervention period less than half a year $(n=340$, SMD $=-0.95,95 \%$ CI -1.64 to $-0.26, P=0.007$ ), or more than one year $(n=371, \mathrm{SMD}=-0.93,95 \% \mathrm{CI}-1.72$ to -0.15 ,
$P=0.02)$. However, their heterogeneities of both comparisons also were substantive with $I^{2}$ value being $87 \%$ and $91 \%$, respectively (Table 5). Sensitivity analysis showed TCC intervention remained significant reduction of LDL-C level at intervention period less than half a year $(n=280$, SMD $=$ $-0.58,95 \%$ CI -0.82 to $-0.33, P=0.0002, I^{2}=29 \%$ ) excluded one study [23], but at intervention period more than one year, after excluding any study, the effect size was remained statistically significant but still indicated high heterogeneity among studies. Two studies $[45,49]$ with 98 patients with chronic disease reported TCC intervention plus conventional treatment versus same conventional treatment on LDL-C level, and pooled analysis was not performed because of the high heterogeneity $\left(I^{2}=96 \%\right)$ existed between the two studies. One study [45] reported a significant difference between comparison groups $(n=60, \mathrm{SMD}=-0.54,95 \% \mathrm{CI}$ -0.73 to $-0.35, P<0.00001$ ), and another study [49] reported no significant difference $(n=38, \mathrm{SMD}=-0.05,95 \% \mathrm{CI}-0.13$ to $0.03, P=0.22$ ) (Table 5).

3.4.5. FBG, $P B G, F P I$. 6 studies mainly involving in healthy participants comparing TCC intervention with nonintervention and 2 studies with 64 patients with chronic disease comparing TCC intervention plus conventional treatment with same conventional treatment reported FBG level. Compared to nonintervention, TCC intervention showed a significant reduction of FBG level $(n=230$, SMD $=-0.93,95 \%$ CI -1.42 to $-0.43, P=0.0003, I^{2}=67 \%$ ) (Table 6). Compared with same conventional treatment used alone in patients with chronic disease, TCC combined with conventional treatment did not show a significant difference on FBG level $(n=64$, $\mathrm{MD}=0.60 \mathrm{mmol} / \mathrm{L}, 95 \% \mathrm{CI}-0.94$ to $2.14, P=0.45, I^{2}=$ 73\%) (Table 6).

One study [30] with 32 healthy participants reported the effect of TCC intervention on postprandial two-hour blood glucose (PBG) in comparison to nonintervention, and results showed a significant reduction of PBG level $(\mathrm{MD}=$ $-1.40 \mathrm{mmol} / \mathrm{L}, 95 \% \mathrm{CI}-1.64$ to $-1.16, P<0.0001$ ) (Table 6).

There were two studies $[29,31]$ that reported the effect of TCC intervention on fasting plasma insulin (FPI) in comparison to nonintervention, meat-analysis was not used for significant difference because of a high heterogeneity $\left(I^{2}=\right.$ 91\%), and in this comparison, TCC intervention showed a significant reduction of FPI level $(n=40, \mathrm{MD}=-6.80 \mathrm{U} / \mathrm{L}$, $95 \%$ CI -10.42 to $-3.48, P<0.0001)$ in one study [29] and no significant difference $(n=19, \mathrm{MD}=7.99 \mathrm{U} / \mathrm{L}, 95 \% \mathrm{CI}$ -1.41 to $17.39, P=0.1$ ) in another study [31] (Table 6). One study [56] with 40 patients with chronic disease reported TCC intervention plus conventional treatment versus same conventional treatment, and result showed no significant difference of FPI level ( $\mathrm{MD}=-0.10 \mathrm{U} / \mathrm{L}, 95 \% \mathrm{CI}-0.3$ to 0.10 , $P=0.32$ ) (Table 6).

3.4.6. Adverse Effects. None of included studies reported adverse events. 
TABle 5: The effect of TCC on blood lipids.

\begin{tabular}{|c|c|c|c|c|c|c|}
\hline Outcome or subgroup title & $\begin{array}{c}\text { Number } \\
\text { of } \\
\text { studies }\end{array}$ & $\begin{array}{l}\text { Number of } \\
\text { participants }\end{array}$ & $\begin{array}{l}\text { Effect size; std. mean difference (IV, } \\
\text { random, 95\% CI) }\end{array}$ & $P$ value & $I^{2}$ & $\mathrm{P}_{\text {Heterogeneity }}$ \\
\hline \multicolumn{7}{|c|}{ Tai Chi Chuan versus nonintervention } \\
\hline \multicolumn{7}{|l|}{ TC } \\
\hline Intervention for less than half a year & 7 & 340 & $-0.87[-1.66,-0.08]$ & 0.03 & $90 \%$ & $<0.00001$ \\
\hline Intervention for more than 1 year & 5 & 298 & $-0.32[-0.63,-0.02]$ & 0.04 & $40 \%$ & 0.15 \\
\hline \multicolumn{7}{|l|}{ TG } \\
\hline Intervention for less than half a year & 7 & 340 & $-0.58[-1.03,-0.14]$ & 0.01 & $72 \%$ & 0.002 \\
\hline Intervention for more than 1 year & 5 & 298 & $-0.67[-0.90,-0.43]$ & $<0.00001$ & $0 \%$ & 0.82 \\
\hline \multicolumn{7}{|l|}{ HDL-C } \\
\hline Intervention for less than half an year & 7 & 340 & $0.77[0.01,1.53]$ & 0.05 & $90 \%$ & $<0.00001$ \\
\hline Intervention for more than 1 year & 5 & 335 & $0.88[0.44,1.32]$ & $<0.0001$ & $73 \%$ & 0.006 \\
\hline \multicolumn{7}{|l|}{ LDL-C } \\
\hline Intervention for less than half an year & 7 & 340 & $-0.95[-1.64,-0.26]$ & 0.007 & $87 \%$ & $<0.00001$ \\
\hline Intervention for more than 1 year & 6 & 371 & $-0.93[-1.72,-0.15]$ & 0.02 & $91 \%$ & $<0.00001$ \\
\hline \multicolumn{7}{|c|}{ Tai Chi Chuan plus conventional treatment versus same conventional treatment } \\
\hline $\mathrm{TC}(\mathrm{mmol} / \mathrm{L})$ & 1 & 60 & $-1.76[-2.02,-1.50]$ & & & $<0.00001$ \\
\hline $\mathrm{TG}(\mathrm{mmol} / \mathrm{L})$ & 1 & 60 & $-0.05[-0.27,0.17]$ & & & 0.66 \\
\hline $\mathrm{HDL}-\mathrm{C}(\mathrm{mmol} / \mathrm{L})$ & 1 & 60 & $0.20[0.16,0.24]$ & & & $<0.00001$ \\
\hline \multicolumn{7}{|l|}{$\mathrm{LDL}-\mathrm{C}(\mathrm{mmol} / \mathrm{L})$} \\
\hline Chen 2013 [45] & 1 & 60 & $-0.54[-0.73,-0.35]$ & & & $<0.00001$ \\
\hline Li et al. 2006 [49] & 1 & 38 & $-0.05[-0.13,0.03]$ & & & 0.22 \\
\hline
\end{tabular}

TC: total cholesterol; TG: triglycerides; HDL-C: high-density lipoprotein cholesterol; LDL-C: low-density lipoprotein cholesterol.

TABLE 6: The effect of TCC on fasting blood glucose, postprandial two-hour blood glucose, and fasting plasma insulin.

\begin{tabular}{|c|c|c|c|c|c|c|}
\hline Outcome or subgroup title & $\begin{array}{c}\text { Number } \\
\text { of } \\
\text { studies }\end{array}$ & $\begin{array}{l}\text { Number of } \\
\text { participants }\end{array}$ & $\begin{array}{l}\text { Effect size; mean difference } \\
\quad \text { (IV, fixed, 95\% CI) }\end{array}$ & $P$ value & $I^{2}$ & $\mathrm{P}_{\text {Heterogeneity }}$ \\
\hline \multicolumn{7}{|c|}{ Tai Chi Chuan versus non-intervention } \\
\hline FBG & 6 & 230 & $-0.93[-1.42,-0.43]^{\star}$ & 0.0003 & $67 \%$ & 0.01 \\
\hline $\mathrm{PBG}(\mathrm{mmol} / \mathrm{L})$ & 1 & 32 & $-1.40[-1.64,-1.16]$ & $<0.00001$ & & \\
\hline \multicolumn{7}{|l|}{ FPI } \\
\hline Wu et al. 2010 (U/L) [29] & 1 & 40 & $-6.80[-10.12,-3.48]$ & $<0.0001$ & & \\
\hline Zhang and $\mathrm{Fu} 2008(\mathrm{pM})$ [31] & 1 & 19 & $7.99[-1.41,17.39]$ & 0.1 & & \\
\hline \multicolumn{7}{|c|}{ Tai Chi Chuan plus conventional treatment versus same conventional treatment } \\
\hline $\mathrm{FBG}(\mathrm{mmol} / \mathrm{L})$ & 2 & 64 & $0.60[-0.94,2.14]$ & 0.45 & $73 \%$ & 0.05 \\
\hline FPI (U/L) & 1 & 40 & $-0.10[-0.30,0.10]$ & 0.32 & & \\
\hline
\end{tabular}

Std: mean difference (IV, random, 95\% CI).

FBG: fasting blood glucose; PBG: postprandial two-hour blood glucose; FPI: fasting plasma insulin.

\section{Discussion}

A comprehensive search was conducted through major electronic databases for interventions involving in TCC exercise. Reference lists of systematic review were also screened when necessary. All screening of eligible studies, data extraction and analysis were carried out independently by two review authors. Our decision to restrict the investigated interventions to comparing TCC exercise or/plus conventional treatment with nonintervention/or same conventional treatment in this review avoided the potential confounding effects of other behavioral interventions on the outcomes, such as those involving other exercises, different dietary interventions or interventions that focused on weight loss. In this systematic review, 36 studies accounting for 2393 participants (1094 healthy adults and 1299 patients with chronic diseases) were identified. Only 4 out of 23 included RCTs reported definitive randomization, 2 studies were selfcontrolled trials, and the remaining trials were controlled trials without randomization. We attempted to contact 
TABLE 7: Characteristics of excluded studies [ordered by study ID].

\begin{tabular}{|c|c|}
\hline Study & Reason for exclusion \\
\hline Bi and Chen, $2005^{[1]}$ & No outcomes of interest \\
\hline Chang et al., $2013^{[2]}$ & Frequency of intervention is less than 3 times a week \\
\hline Channer et al., $1996^{[3]}$ & No data for extraction \\
\hline Chang et al., $2013^{[4]}$ & No data for extraction \\
\hline Fu and Guo, $2013^{[5]}$ & No data for extraction \\
\hline Rosado-Perez et al., 2012 $2^{[6]}$ & No data for extraction \\
\hline Lam et al., $2008^{[7]}$ & Frequency of intervention is less than 3 times a week \\
\hline Motivala et al., $2006^{[8]}$ & Not parallel control design \\
\hline Nguyen and Kruse, $2010^{[9]}$ & Frequency of intervention is less than 3 times a week \\
\hline Thomas et al., $2005^{[10]}$ & No data for extraction \\
\hline Thornton and Tang, 2004 $4^{[11]}$ & No data for extraction \\
\hline Wang, $2010^{[12]}$ & No data for extraction \\
\hline Wang et al., $2004^{[13]}$ & Not parallel control design \\
\hline Wolf et al., $2003^{[14]}$ & Not parallel control design \\
\hline Wolf et al., $2006^{[15]}$ & Frequency of intervention is less than 3 times a week \\
\hline Zhang and Tan, $2006^{[16]}$ & No data for extraction \\
\hline
\end{tabular}

References to studies excluded from this review:

${ }^{[1]}$ Y. Bi and W. H. Chen, "The effects of Tai Chi exercise on blood rheology in patients with hypertension," Chinese Journal of Sports Medicine, vol. 24, pp. 606607, 2005.

${ }^{[2]}$ R. Y. Chang, M. Koo, C. K. Chen, Y. C. Lu and Y. F. Lin, "Effects of habitual T'ai Chi exercise on adiponectin, glucose homeostasis, lipid profile, and atherosclerotic burden in individuals with cardiovascular risk factors," Journal of Alternative and Complementary Medicine, vol. 19, pp. 697-703, 2013.

${ }^{[3]}$ K. S. Channer, D. Barrow, R. Barrow, M. Osborne, and G. Ives, "Changes in haemodynamic parameters following Tai Chi Chuan and aerobic exercise in patients recovering from acute myocardial infarction," The Fellowship of Postgraduate Medicine, vol. 72, pp. 349-351, 1996.

${ }^{[4]}$ M.-Y. Chang, S.-C. J. Yeh, M.-C. Chu et al., "Associations between Tai Chi Chung Program, Anxiety, and Cardiovascular Risk Factors," Am J Health Promot, vol. 28, pp. 16-22, 2013.

${ }^{[5]} \mathrm{X}$. Fu and J. Guo, "The study of the influence of Tai Chi exercise on physical fitness and its composition of the middle-aged and eldly," Journal of Gansu Normal Colleges, vol. 14, pp. 73-76, 2009.

${ }^{[6]}$ J. Rosado-Perez, E. Santiago-Osorio, R. Ortiz et al., "Tai Chi diminishes oxidative stress in Mexican older adults," Journal of Nutrition, Health and Aging, vol. 16, pp. 642-646, 2012.

${ }^{[7]}$ P. Lam, S. M. Dennis, T. H. Diamond et al., "Improving glycemic and BP control in type 2 diabetes: The effectiveness of Tai Chi," Australian Family Physician, vol. 37, pp. 884-887, 2008.

${ }^{[8]}$ S. J. Motivala, J. Sollers, J. Thayer et al., "Tai Chi Chih acutely decreases sympathetic nervous system activity in older adults," Journals of Gerontology: Series A Biological Sciences and Medical Sciences, vol. 61, pp. 1177-1180, 2006.

${ }^{[9]}$ M. H. Nguyen and R. Kruse, "The effects of Tai Chi training on physical fitness, perceived health, and blood pressure in elderly Vietnamese," Open Access Journal of Sports Medicine, vol. 2012, pp. 7-16, 2012.

${ }^{[10]}$ G. N. Thomas, W. L. Hong Athena, B. Tomlinson et al., "Effects of Tai Chi and resistance training on cardiovascular risk factors in elderly Chinese subjects: a 12-month longitudinal, randomized, controlled intervention study," Clin Endocrinol (Oxf), vol. 63, pp. 663-669, 2005.

${ }^{[11]}$ E. W. Thornton, K. S. Sykes, and W. K. Tang, "Health benefits of Tai Chi exercise: Improved balance and blood pressure in middle-aged women," Health Promotion International, vol. 19, pp. 33-38, 2004.

${ }^{[12]}$ G. J. Wang, "The effects of Tai Chi exercise on cardiopulmonary function in the elderly," Chinese Journal of Gerontology, p. 12, 2010.

${ }^{[13]}$ Y. G. Wang, G. F. Lv, and Y. B. Ren, "The effects of exercise therapy on type 2 diabetes in the middle-aged and elderly," Chinese Journal of Sports Medicine, vol. 23, pp. 679-681, 2004.

${ }^{[14]}$ S. L. Wolf, X. Barnhart Huimnan, Kutner, G. Nancy et al., "Selected as the Best Paper in the 1990s: Reducing Frailty and Falls in Older Persons: An Investigation of Tai Chi and Computerized Balance Training," Journal of the American Geriatrics Society, vol. 51, pp. 1794-1803, 2003.

${ }^{[15]}$ S. L. Wolf, M. O'Grady, K. A. Easley et al., "The influence of intense Tai Chi training on physical performance and hemodynamic outcomes in transitionally frail, older adults," Journals of Gerontology: Series A Biological Sciences and Medical Sciences, vol. 61, pp. 184-189, 2006.

${ }^{[16]}$ T. M. Zhang and Y. M. Tan, "The effects of Tai Chi on fitness in middle-aged and elderly women comparing with the young," Chinese Journal of Clinical Rehabilitation, vol. 10, pp. 76-78, 2006.

authors by telephone or e-mail for further information. But most replies were unsatisfactory and did not resolve our questions even no response. Therefore, as a whole, the majority of the included studies belonged to low methodological quality. Of all studies, 22 studies were designed to compare TCC with nonintervention [23-44]. The remaining studies were designed to compare TCC plus conventional treatment with the same conventional treatment.
In the primary outcomes, two studies with 185 participants assessed incidence of nonfatal, fatal stroke, cardiacerebrovascular disease (CCD), or cardiac failure after five years' follow-up period, their results showed TCC exercise had a beneficial effects on preventing the incidence of nonfatal stroke and CCD. However, because of insufficient data, we cannot make any conclusion about the effects of TCC preventing the occurrence of stroke directly, more trials 


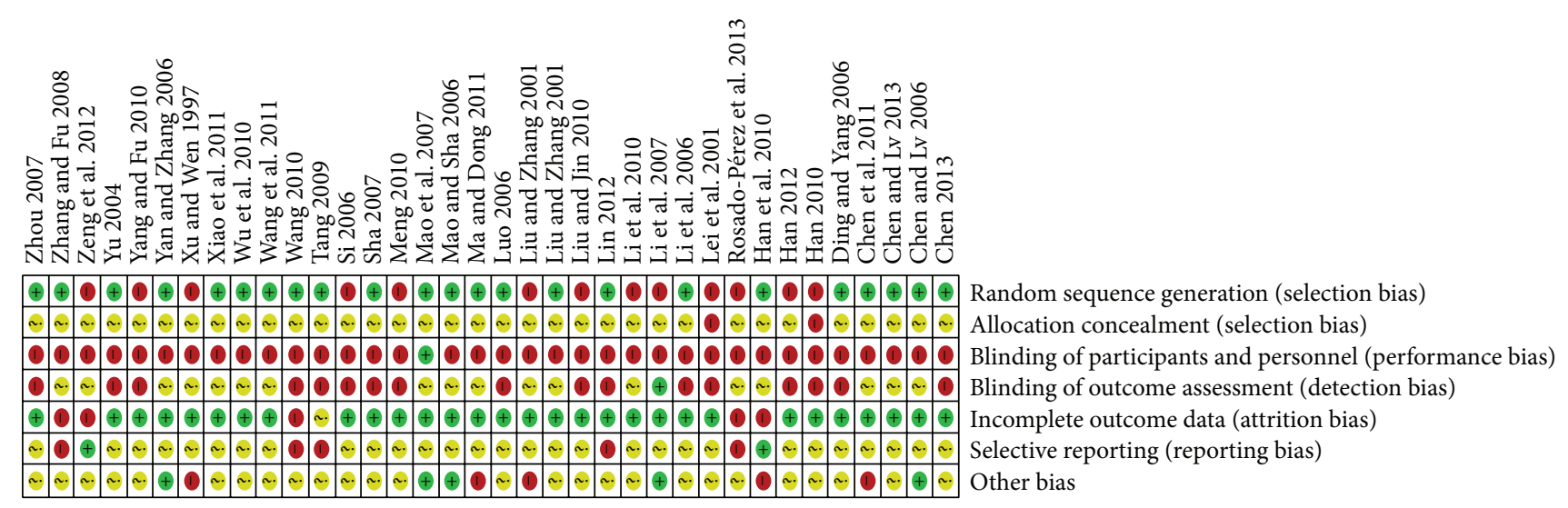

FIGURE 3: "Risk of bias" summary: review authors' judgments about each risk of bias each included study.

which investigated the effects of TCC on the clinic outcomes of stroke with long follow-up period are need. Nonetheless, in this review we also examined secondary outcomes mainly including the risk factors of stroke such as body weight, blood pressure, lipid levels and blood glucose and so on. In the present review, compared with nonintervention, TCC intervention remarkably lowered body weight, BMI, SBP, DBP and FBG on healthy adults; It also significantly decreased plasma TC, TG, and LDL-C level, and raised HDL-C level. Compared with other treatment (conventional treatment or health education), TCC intervention on the basis of same other treatment could significantly lower SBP, DBP and plasma LDL-C level in patients with chronic diseases. The results showed the comprehensive controlled effect of TCC intervention on risk factors of stroke and indicated that it might be effective in preventing stroke in healthy adults or patients with chronic diseases. Because small reductions in risk factors of stoke may lead to larger reductions in stoke incidence throughout a whole population [59]. Nevertheless, concluding that TCC have definitively preventive effects on stoke would be premature because most of the studies were of low methodological quality with obvious design shortcomings such as inadequate concealment, no blinding, and incomplete outcomes data point to the possibility of bias. Additionally, clinical heterogeneity was apparent because different style of TCC was used.

Up to present, some systematic reviews have looked at TCC for cancer, balance, sleep quality, osteoarthritis, fibromyalgia and rheumatoid arthritis [60-65], or have look at TCC for the rehabilitation of stroke [66]. We did not find other systematic review had been carried out to examine the effects of TCC for the primary prevention of stroke in adults. Other reviews have examined the effects of TCC for blood pressure, blood lipid, and blood glucose on healthy old population or patients with chronic disease $[19,20,67,68]$. Their results indicated limited evidence for the effectiveness of TCC on blood pressure and blood lipid other than blood glucose in the elderly. One review investigated the effect of TCC for the primary prevention of CVD, and found some suggestions of beneficial effects of TCC on CVD risk factors, but this review was based on only 13 trials with a small sample sizes and the conclusion cannot be drawn because of inconsistency across all included trials [69]. In present review, we analyzed 36 studies accounting for 2393 participants, the results shown that TCC exercise had the significant benefit in modifying the risk factors of stoke in middle-aged and elderly adults such as body weight, blood pressure, lipid levels and blood glucose. Researches have demonstrated stroke can be prevented through controlling its modifiable risk factors, and small reductions of risk factors may lead to larger decrease in the incidence of stroke throughout a whole population [8-10]. Accordingly, we thought that TCC exercise might be beneficial for the primary prevention of stroke by modifying the risk factors. Furthermore few studies in present review also found that TCC with a long term training significantly decreased the incidence of nonfatal stroke $[48,58]$. Therefore, there was some suggestion of beneficial effects of TCC exercise on the primary prevention of stroke but large longterm trials are needed to confirm this evidence.

Several limitations were identified in this review. The key limitation was quality of the included studies. Mostly included studies were of low quality due to no definite on random sequence generation, allocation concealment, and the blinding outcome assessors. The potential bias might erode the reliability of conclusions of this review. Secondly, the direct effect of TCC preventing the occurrence of stroke cannot be assessed due to no enough follow-up period performed in the included studies. Thirdly, funnel plot analysis could not be conducted due to the insufficient number of studies included for any outcomes in this review, however, majority of included studies published in China. Therefore the possible publication bias is inevitable. In addition, the variations on the characteristics of participants and intervention period indicated some clinical heterogeneity among the included trials.

In this current review, few studies evaluate TCC for the primary preventive effect of stroke by examining the direct outcomes, but the results suggest that TCC exercise may be effective in modifying the risk factors of stroke. Due to the limitation of methodological quality and the limited evidence available, currently no conclusion can be drawn as to the effectiveness of TCC exercise for the primary prevention 
TABLE 8: Checklist of the PRISMA statement on systematic review.

\begin{tabular}{llrl}
\hline Section/topic & Item Checklist item & Reported on page \\
\hline $\begin{array}{l}\text { Title } \\
\text { Title }\end{array}$ & $1 \quad$ Identify the report as a systematic review, meta-analysis, or both. & Title (Page 1)
\end{tabular}

Abstract

Structured summary

Introduction

Rationale

3 Describe the rationale for the review in the context of what is already known.
Provide a structured summary including, as applicable: background; objectives; data sources; study eligibility criteria, participants, and

2 interventions; study appraisal and synthesis methods; results; limitations; conclusions and implications of key findings; systematic review registration number.
Abstract

(Page 1-2)
Title

Objectives

Methods

Protocol and registration

Eligibility criteria

Information sources

Search

Study selection

Data collection process

Data items

Risk of bias in individual studies

Summary measures

Synthesis of results

Risk of bias across studies

Additional analyses

Results

Study selection

Title

Study characteristics

Risk of bias within studies
Provide an explicit statement of questions being addressed with reference to 4 participants, interventions, comparisons, outcomes, and study design (PICOS).

Indicate if a review protocol exists, if and where it can be accessed (e.g., Web

5 address), and, if available, provide registration information including registration number.

Specify study characteristics (e.g., PICOS, length of follow-up) and report

6 characteristics (e.g., years considered, language, publication status) used as criteria for eligibility, giving rationale.

Describe all information sources (e.g., databases with dates of coverage,

7 contact with study authors to identify additional studies) in the search and date last searched.

Introduction

(Page 2-3)

Methods

Present full electronic search strategy for at least one database, including any limits used, such that it could be repeated.

9 State the process for selecting studies (i.e., screening, eligibility, included in systematic review, and, if applicable, included in the meta-analysis).

Describe method of data extraction from reports (e.g., piloted forms,

10 independently, in duplicate) and any processes for obtaining and confirming data from investigators.

11

List and define all variables for which data were sought (e.g., PICOS, funding sources) and any assumptions and simplifications made.

Describe methods used for assessing risk of bias of individual studies

12 (including specification of whether this was done at the study or outcome level), and how this information is to be used in any data synthesis.

13 State the principal summary measures (e.g., risk ratio, difference in means).

14 Describe the methods of handling data and combining results of studies, if done, including measures of consistency (e.g., $I^{2}$ ) for each meta-analysis.

15 Specify any assessment of risk of bias that may affect the cumulative evidence (e.g., publication bias, selective reporting within studies).

16

Describe methods of additional analyses (e.g., sensitivity or subgroup analyses, metaregression), if done, indicating which were prespecified.

17 Give numbers of studies screened, assessed for eligibility, and included in the review, with reasons for exclusions at each stage, ideally with a flow diagram.

Figure 1

Table 1

18 For each study, present characteristics for which data were extracted (e.g., study size, PICOS, follow-up period) and provide the citations.

N/A

Methods

Methods

(Page 3)

Appendix 1

Methods

(Page 4)

Methods

(Page 4)

Methods

(Page 4)

Methods

(Page 4)

Methods

(Page 4)

Methods

(Page 4)

N/A

Methods

(Page 4)

Present data on risk of bias of each study and, if available, any outcome-level assessment (see Item 12). 
TABLE 8: Continued.

\begin{tabular}{|c|c|c|c|}
\hline Section/topic & Item & Checklist item & Reported on page \\
\hline Results of individual studies & 20 & $\begin{array}{l}\text { For all outcomes considered (benefits or harms), present, for each study: (a) } \\
\text { simple summary data for each intervention group and (b) effect estimates and } \\
\text { confidence intervals, ideally with a forest plot. }\end{array}$ & Tables 2-6 \\
\hline Synthesis of results & 21 & $\begin{array}{l}\text { Present results of each meta-analysis done, including confidence intervals and } \\
\text { measures of consistency. }\end{array}$ & $\mathrm{N} / \mathrm{A}$ \\
\hline Risk of bias across studies & 22 & Present results of any assessment of risk of bias across studies (see Item 15). & $\mathrm{N} / \mathrm{A}$ \\
\hline Additional analysis & 23 & $\begin{array}{l}\text { Give results of additional analyses, if done (e.g., sensitivity or subgroup } \\
\text { analyses, metaregression [see Item 16]). }\end{array}$ & $\begin{array}{l}\text { Results } \\
\text { (Page 8-10) }\end{array}$ \\
\hline \multicolumn{4}{|l|}{ Discussion } \\
\hline Summary of evidence & 24 & $\begin{array}{l}\text { Summarize the main findings including the strength of evidence for each } \\
\text { main outcome; consider their relevance to key groups (e.g., health care } \\
\text { providers, users, and policy makers). }\end{array}$ & $\begin{array}{l}\text { Discussion } \\
\text { (Page 13) }\end{array}$ \\
\hline Limitations & 25 & $\begin{array}{l}\text { Discuss limitations at study and outcome level (e.g., risk of bias), and at review } \\
\text { level (e.g., incomplete retrieval of identified research, reporting bias). }\end{array}$ & $\begin{array}{l}\text { Discussion } \\
\text { (Page 13) }\end{array}$ \\
\hline Conclusions & 26 & $\begin{array}{l}\text { Provide a general interpretation of the results in the context of other evidence, } \\
\text { and implications for future research. }\end{array}$ & $\begin{array}{l}\text { Discussion } \\
\text { (Page 13-14) }\end{array}$ \\
\hline \multicolumn{4}{|l|}{ Funding } \\
\hline Funding & 27 & $\begin{array}{l}\text { Describe sources of funding for the systematic review and other support (e.g., } \\
\text { supply of data); role of funders for the systematic review. }\end{array}$ & $\begin{array}{l}\text { Acknowledgments } \\
\text { (Page 14) }\end{array}$ \\
\hline
\end{tabular}

of stroke. Large prospective long-term trials with rigorous design are needed before TCC exercise can be recommended as a population-based intervention to prevent the incidence of stroke.

\section{Appendices}

\section{A. Search Strategies}

A.1. Science Citation Index (SCI)

\#1 TS: (stroke)

\#2 TS: (“Cerebral hemorrhage")

\#3 TS: (Infarction)

\#4 TS: (Hemiplegia)

\#5 TS: (“Blood pressure")

\#6 TS: ("Systolic blood pressure”)

\#7 TS: ("Diastolic blood pressure")

\#8 TS: (Cholesterol)

\#9 TS: (Triglycerides)

\#10 TS: ("lipoproteins, ldl”)

\#11 TS: (“lipoproteins, hdl”)

\#12 TS: (“Blood sugar”)

\#13 \#1-\#12/OR

\#14 TS: (“Tai ji”)

\#15 TS: (Taiji)

\#16 TS: (“Tai-ji”)

\#17 TS: (“Tai Chi”)

\#18 TS: (“Chi, Tai”)

\author{
\#19 TS: (“Tai Ji Quan”) \\ \#20 TS: (“Ji Quan, Tai”) \\ \#21 TS: (“Quan, Tai Ji”) \\ \#22 TS: (Taijiquan) \\ \#23 TS: (“T’ai Chi”) \\ \#24 TS: (“Tai Chi Chuan”) \\ \#25 \#14-\#24/OR \\ \#26 TS: (control) \\ \#27 TS: (Comparison) \\ \#28 TS: (“Controlled trial”) \\ \#29 \#26 OR \#27 OR \#28 \\ \#29 \#13 AND \#25 AND \#29.
}

\section{A.2. PubMed}

\#1 stroke [MeSH Terms]

\#2 Cerebral hemorrhage [MeSH Terms]

\#3 Infarction [MeSH Terms]

\#4 Hemiplegia [MeSH Terms]

\#5 Blood pressure [MeSH Terms]

\#6 "Systolic blood pressure" [ALL]

\#7 “Diastolic blood pressure”[ALL]

\#8 Cholesterol [MeSH Terms]

\#9 Triglycerides [MeSH Terms]

\#10 lipoproteins, ldl [MeSH Terms]

\#11 lipoproteins, hdl [MeSH Terms]

\#12 Blood sugar [MeSH Terms] 


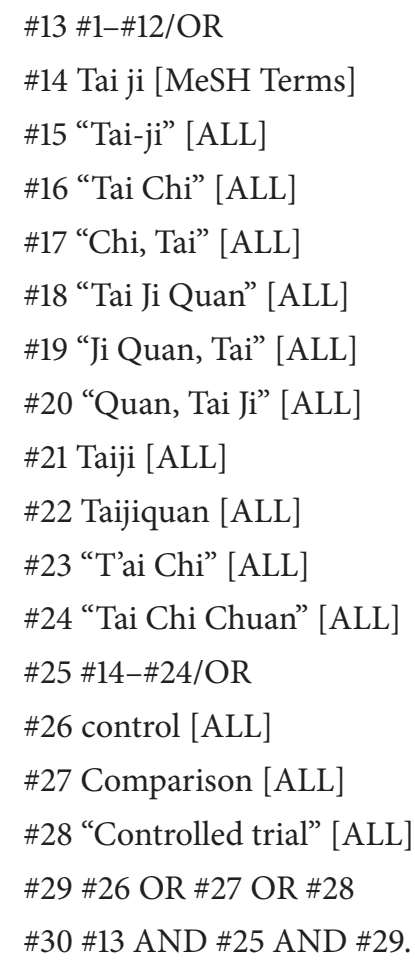

\section{A.3. The Cochrane Library}

\#1 MeSH descriptor: [Stroke] expllode all trees

\#2 stroke or strokes

\#3 cerebrovasc*

\#4 cerebral next vascular

\#5 apoplexy

\#6 brain near/2 accident*

\#7 brain* near/2 infarct*

\#8 cerebral near/2 infarct*

\#9 lacunar near/2 infarct*

\#10 MeSH descriptor: [Hypertension] explode all trees

\#11 hypertensi*

\#12 peripheral next arter* next disease*

\#13 high near/2 blood next pressure

\#14 increased near/2 blood next pressure

\#15 elevated near/2 blood next pressure

\#16 MeSH descriptor: [Hyperlipidemias] explode all trees

\#17 hyperlipid*

\#18 hyperlip?emia*

\#19 hypercholesterol ${ }^{*}$

\#20 hypercholester?emia*

\#21 hyperlipoprotein?emia ${ }^{*}$

\#22 hypertriglycerid?emia*
\#23 MeSH descriptor: [Cholesterol] explode all trees \#24 cholesterol

\#25 MeSH descriptor: [Blood Pressure] this term only \#26 "blood pressure"

\#27 \#1-\#26/or

\#28 MeSH descriptor: [Tai ji] explode all trees

\#29 “Tai-ji”

\#30 “Tai Chi”

\#31 “Chi, Tai”

\#32 “Tai Ji Quan”

\#33 "Ji Quan, Tai”

\#34 "Quan, Tai Ji”

\#35 Taiji

\#36 Taijiquan

\#37 “T'ai Chi”

\#38 “Tai Chi Chuan”

\#39 \#28-\#38/or

\#40 control

\#41 Comparison

\#42 "Controlled trial”

\#43 \#40 or \#41 or \#42

\#44 \#27 and \#39 and \#42.

\section{A.4. Embase Ovid}

\#1 exp Stroke/

\#2 (stroke or stokes).tw.

\#3 cerebrovasc ${ }^{*}$.tw.

\#4 “cerebral vascular".tw.

\#5 apoplexy.tw.

\#6 (brain adj2 accident ${ }^{*}$ ).tw.

\#7 ((brain ${ }^{*}$ or cerebral or lacunar) adj2 infarct $\left.{ }^{*}\right)$.tw.

\#8 exp Hypertension/

\#9 hypertensi ${ }^{*}$.tw.

\#10 “peripheral arter* disease*".tw.

\#11 ((high or increased or elevated) adj2 blood pressure).tw.

\#12 exp Hyperlipidemias/

\#13 hyperlipid*.tw.

\#14 hyperlip?emia* .tw.

\#15 hypercholesterol*.tw.

\#16 hypercholester?emia*.tw.

\#17 hyperlipoprotein?emia ${ }^{*}$.tw.

\#18 hypertriglycerid?emia* ${ }^{*}$.tw.

\#19 exp Arteriosclerosis/ 


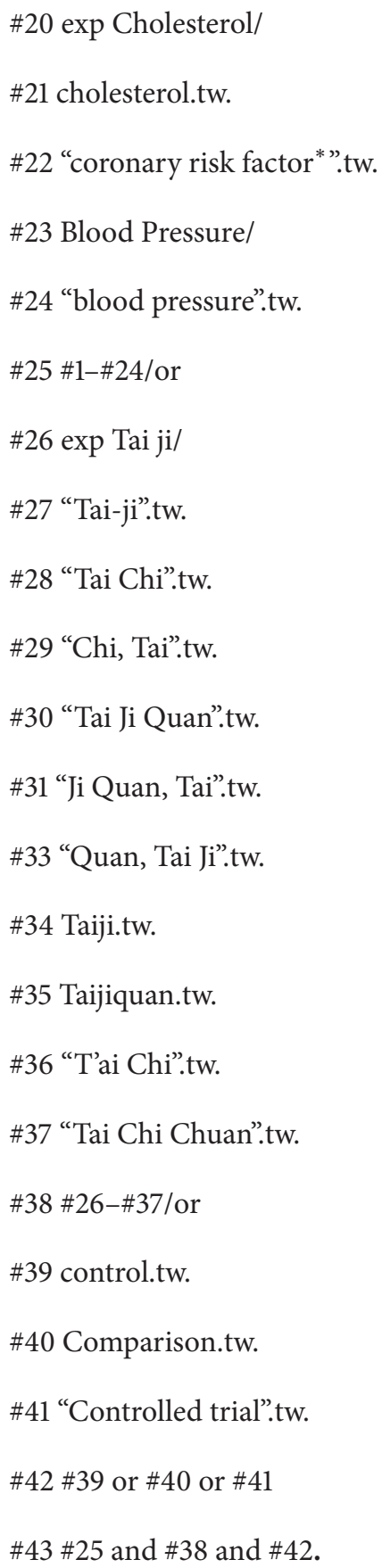

\section{B. Characteristics of Excluded Studies}

See Table 7.

\section{C. "Risk of Bias" Summary}

See Figure 3.

\section{Check list of the PRISMA Statement on Systematic Review}

See Table 8.

\author{
Abbreviations and Acronyms \\ BMI: body mass index \\ CCD: cardia-cerebrovascular disease \\ CVD: cardio vascular disorder \\ DBP: diastolic blood pressure \\ EH: essential hypertension \\ FBG: fasting blood glucose \\ FPI: fasting plasma insulin \\ HDL-C: high-density lipoprotein cholesterol \\ LDL-C: low-density lipoprotein cholesterol \\ NRCT: prospective nonrandomized controlled trial \\ PBF: postprandial two-hour blood glucose \\ RCT: randomized controlled trial \\ SBP: $\quad$ systolic blood pressure \\ TC: $\quad$ total cholesterol \\ TCC: Tai Chi Chuan \\ TG: triglycerides \\ WHR: waist-hip rate.
}

\section{Conflict of Interests}

The authors declare no conflict of interests.

\section{Authors' Contribution}

Guohua Zheng and Maomao Huang are equal contributors.

\section{Acknowledgments}

This systematic review is supported by the Collaboration Innovation Center for Rehabilitation Technology (no. 1 Collaboration Center [2012]) and the Rehabilitation Research Center for Traditional Chinese Medicine, State Administration of Traditional Chinese Medicine of China.

\section{References}

[1] American Stroke Association, "Impact of Stroke," November 2013, http://www.strokeassociation.org.

[2] M. L. Rao, "Guidelines to prevent and control cerebrovascular disease in China," Journal of Apoplexy and Nervous Diseases. In press.

[3] G. X. Wu, Z. S. Wu, J. Liu et al., "Trends of stroke events during 15 years in Beijing (WHO-SINO-MONICA Project Studies)," Chinese Journal of Prevention and Control of Chronic NonCommunicable Diseases, vol. 9, pp. 106-108, 2001.

[4] V. L. Roger, A. S. Go, D. M. Lloyd Jones et al., "Heart disease and stroke statistics-2011 update: a report from the American Heart association," Circulation, vol. 123, no. 4, pp. e18-e219, 2011.

[5] J. J. Caro, K. F. Huybrechts, and I. Duchesne, "Management patterns and costs of acute ischemic stroke: an International Study," Stroke, vol. 31, no. 3, pp. 582-590, 2000.

[6] Ö. Saka, A. Mcguire, and C. Wolfe, "Cost of stroke in the United Kingdom," Age and Ageing, vol. 38, no. 1, pp. 27-32, 2009.

[7] L. B. Goldstein, R. Adams, K. Becker et al., "Primary prevention of ischemic stroke: a statement for healthcare professionals from the stroke council of the American Heart Association," Stroke, vol. 32 , no. 1 , pp. 280-299, 2001 
[8] S. Mittal, The Metabolic Syndrome in Clinical Practice, Springer, London, UK, 2008.

[9] K. J. Egan, H. Janssen, E. S. Sena et al., "Exercise reduces infarct volume and facilitates neurobehavioral recovery: results from a systematic review and meta-analysis of exercise in experimental models of focal ischemia," Neurorehabilitation and Neural Repair, vol. 28, no. 8, pp. 800-812, 2014.

[10] American Heart Association (AHA), "Physical activity improves quality of life," 2013, http://www.heart.org/.

[11] J. Zhou, L. Zhang, H. Liu, T. Mallampati, M. Xu, and J. Yang, "Tai Chi for type 2 diabetes mellitus," The Cochrane Database Systematic Reviews, vol. 3, Article ID CD009717, 2012.

[12] C. Lan, S.-Y. Chen, J.-S. Lai, and M.-K. Wong, "Heart rate responses and oxygen consumption during tai chi chuan practice," The American Journal of Chinese Medicine, vol. 29, pp. 403410, 2001.

[13] K. M. Chen, Y. C. Hsu, W. T. Chen, and H.-F. Tseng, "Well-being of institutionalized elders after Yang-style Tai Chi practice," Journal of Clinical Nursing, vol. 16, no. 5, pp. 845-852, 2007.

[14] A. P. Verhagen, M. Immink, A. van der Meulen, and S. M. A. Bierma-Zeinstra, "The efficacy of Tai Chi Chuan in older adults: a systematic review," Family Practice, vol. 21, no. 1, pp. 107-113, 2004.

[15] H.-M. Lo, C.-Y. Yeh, S.-C. Chang, H.-C. Sung, and G. D. Smith, "A Tai Chi exercise programme improved exercise behaviour and reduced blood pressure in outpatients with hypertension," International Journal of Nursing Practice, vol. 18, no. 6, pp. 545551, 2012.

[16] E. W. Thornton, K. S. Sykes, and W. K. Tang, "Health benefits of Tai Chi exercise: improved balance and blood pressure in middle-aged women," Health Promotion International, vol. 19, no. 1, pp. 33-38, 2004.

[17] W.-A. Lu and C.-D. Kuo, "Three months of Tai Chi Chuan exercise can reduce serum triglyceride and endothelin-1 in the elderly," Complementary Therapies in Clinical Practice, vol. 19, no. 4, pp. 204-208, 2013.

[18] X. Liu, Y. D. Miller, N. W. Burton, J.-H. Chang, and W. J. Brown, "The effect of Tai Chi on health-related quality of life in people with elevated blood glucose or diabetes: a randomized controlled trial," Quality of Life Research, vol. 22, no. 7, pp. 17831786, 2013.

[19] M. S. Lee, T. Y. Choi, H. J. Lim, and E. Ernst, "Tai chi for management of type 2 diabetes mellitus: a systematic review," Chinese journal of integrative medicine, vol. 17, no. 10, pp. 789793, 2011.

[20] C.-L. Lin, C.-P. Lin, and A. S.-Y. Lien, “The effect of tai chi for blood pressure, blood sugar, blood lipid control for patients with chronic diseases: a systematic review," Journal of Nursing, vol. 60, no. 1, pp. 69-77, 2013.

[21] J. Higgins and S. Green, Cochrane Handbook for Systematic Reviews of Interventions, edited by J. P. T. Higgins and S. Green, The Cochrane Collaboration, 2009.

[22] J. P. T. Higgins, S. G. Thompson, J. J. Deeks, and D. G. Altman, "Measuring inconsistency in meta-analyses," British Medical Journal, vol. 327, no. 7414, pp. 557-560, 2003.

[23] Z. Ding and T. Yang, "Effects of Tai Ji quan exercise on blood lipid and lipoprotein metabolism in patients with hyperlipidemia," Chinese Journal of Clinical Rehabilitation, vol. 10, pp. 172-173, 2006.

[24] T. M. Liu and Y. L. Zhang, "Effects of Tai Chi on plasma lipoprotein and blood rheology in the elderly," Modern Rehabilitation, vol. 5, pp. 117-118, 2001.
[25] Z. J. Ma and B. Dong, "Analysis of effects of Tai Chi on blood lipids in the elderly," Sports World, vol. 9, pp. 67-69, 2011.

[26] Y. Mao, L. J. Ma, Y. A. Lv et al., "Effects of Tai Chi and comprehensive exercise on blood lipid metabolism in the older women," Chinese Women's and Children's Health Research, vol. 5, pp. 374-376, 2007.

[27] P. Sha, "Tai Chi exercise intervention on the levels of NO and lipid s in elderly patients with atherosclerosis," Chinese Tissue Engineering Research and Clinical Rehabilitation, vol. 11, pp. 6832-6834, 2007.

[28] K. H. Wang, "Effects of 18-week Tai Chi training on physical function in middle-aged and elderly women," Journal of Xianning College, vol. 30, pp. 103-104, 2010.

[29] F. Wu, E. F. Song, Y. Bao et al., "Effects of 24 style Tai Chi on inflammatory cytokines in serum and the quality of life in patients with type 2 diabetic," Chinese Journal of Physical Medicine and Rehabilitation, vol. 3, pp. 205-207, 2010.

[30] W. Yan and J. Zhang, "Effects of Tai Ji quan exercise on blood sugar and blood lipids in patients with diabetes," Journal of Jiangxi Medical College, vol. 46, pp. 194-195, 2006.

[31] Y. Zhang and F. H. Fu, "Effects of 14-week Tai Ji quan exercise on metabolic control in women with type 2 diabetes," The American Journal of Chinese Medicine, vol. 36, no. 4, pp. 647-654, 2008.

[32] S. W. Zhou, "Effects of Tai Chi exercise on blood pressure and blood lipids in patients with type 1 essential hypertension," in International Medical Guide, vol. 13, pp. 60-64, 2007.

[33] Y. Z. Han, Phase Evaluation Study of Tai Chi Exercise on Cardiopulmonary Function and Balance in the Elderly, Northeast Normal University, 2010.

[34] X. S. Lei, H. Y. Ni, Q. Y. Chen et al., "Effects of 42 style Tai Chi on cardiovascular function and respiratory endurance in the middle-aged and elderly," Modern Rehabilitation, vol. 11, pp. 64-65, 2001.

[35] Y. B. Han, "Effects of 12-week Tai Chi training on serum adiponectin levels in postmenopausal women," Chinese Journal of Gerontology, vol. 32, pp. 38-41, 2012.

[36] J. Rosado-Pérez, R. Ortiz, E. Santiago-Osorio, and V. M. Mendoza-Núñez, "Effect of Tai Chi versus walking on oxidative stress in Mexican older adults," Oxidative Medicine and Cellular Longevity, vol. 2013, Article ID 298590, 8 pages, 2013.

[37] C. W. Li, Y. Zeng, and F. L. Peng, "Changes in vascular endothelial diastolic function in the elderly and the effects of practicing Tai Chi," Journal of Clinical Rehabilitative Tissue Engineering Research, vol. 11, pp. 5414-5416, 2007.

[38] G. P. Li, Y. C. Fang, Y. J. Zhang et al., "Effects of Tai Chi exercise on physical and mental health of the middle-aged and elderly," Journal of Nursing, vol. 2, pp. 5-7, 2010.

[39] X. D. Liu and H. Z. Jin, "Observation on effects of Tai Chi exercise on cardiopulmonary function in the elderly," Practical Chinese Medicine, vol. 5, pp. 34-35, 2010.

[40] T. M. Liu and Y. L. Zhang, "Effects of Tai Ji Quan on plasma lipoprotein and blood rheology in the elderly," Modern Rehabilitation, vol. 5, pp. 117-118, 2001.

[41] X. T. Meng, "Effects of Tai Ji quan exercise on antioxidant capacity in middle-aged and elderly women," Electronic Journals of Martial Arts and Sciences, vol. 5, pp. 39-40, 2010.

[42] C. J. Si, "Effects of Tai Ji quan exercise on physical fitness in the middle-aged and elderly," Journal of Liaoning Normal University (Natural Science Edition), vol. 29, pp. 379-381, 2006.

[43] M. Xu and Z. H. Wen, "Changes of cardiopulmonary function before and after Tai Chi exercise in the elderly," Journal of Chengdu Institute of Physical Education, vol. 3, pp. 80-83, 1997. 
[44] Y. Yang and X. Fu, "Effects of Tai Ji quan exercise on physical fitness in the middle-aged and elderly," Scientific and Technological Information, vol. 28, pp. 271-272, 2010.

[45] J. F. Chen, "Effects of Tai Ji quan exercise on blood lipids in elderly patients with coronary heart disease," Guide of China Medicine, vol. 11, pp. 583-584, 2013.

[46] X. X. Chen and H. Q. Lv, "Effects of Tai Ji quan exercise on NO concentration in plasma, RBC membrane sodium and calcium pump activity in patients with hypertension," Journal of Beijing University of Physical Education, vol. 29, pp. 1359-1361, 2006.

[47] F. Z. Chen and Q. B. Lv, "Effects of Tai Ji quan on blood pressure in patients with hypertension," Contemporary Nurse, vol. 4, pp. $18-19,2013$

[48] Q. Y. Han, X. F. Huang, L. Li, and L. Q. Chen, "Effects of Tai Ji quan exercise on long-term quality of life in middle-aged and elderly patients with essential hypertension," Journal of Chinese Modern Nursing, no. 14, pp. 1617-1619, 2010.

[49] J. Z. Li, D. P. Zhai, C. Qiu et al., "Antioxidant effects of Tai Chi exercise on patients with coronary heart disease," Chinese Journal of Clinical Rehabilitation, vol. 10, pp. 155-156, 2006.

[50] H. Z. Lin, "The clinical effect of Tai Chi exercise combined with irbesartan on patients with early diabetic nephropathy," Chinese National Folk Medicine Magazine, vol. 21, pp. 108-109, 2012.

[51] H. Luo, "Tai Chi combined with drug in treatment of essential hypertension: a clinical study," Chinese Pharmaceutical Herald, vol. 33, pp. 43-44, 2006.

[52] H.-N. Mao and P. Sha, "Effect of Tai Chi exercise on blood pressure, plasma nitrogen monoxidum and endothelin in hypertensive patients," Chinese Journal of Clinical Rehabilitation, vol. 10, no. 48, pp. 65-67, 2006.

[53] Q. H. Tang, "Effects of traditional sports on clinical symptoms of aged intellectuals with essential hypertension: an observational study," Journal of Beijing University of Physical Education, vol. 2, pp. 67-69, 2009.

[54] X. J. Wang, Y. J. Li, and N. N. Liu, “Tai Chi exercise intervention on the prevention and treatment of hypertension: an empirical study," Journal of Beijing University of Physical Education, vol. 34, pp. 75-77, 2011.

[55] L. Xiao, Y. Zhou, and J. Li, "Effects of Tai Chi exercise and puerarin intervention on the leve of FBG and Serum NO and NOS activity," Journal of Shaanxi Normal University: Natural Science, vol. 39, pp. 104-108, 2011.

[56] Y. Yu, "Study on the mechanism of effects of Tai Chi exercise on patients with type 2 diabetes accompanied hypertension," in Proceedings of the Beijing International Exercise Physiology Thesis Presentation, 2004.

[57] J. Y. Chen, J. H. Li, W. J. Chen et al., "Effect evaluation of Tai Chi intervention on hypertension in Liu Rong community of Guangzhou," Medical Information, vol. 24, pp. 13-14, 2011.

[58] Y. H. Zeng, Y. P. Zeng, L. Li et al., "Effects of long-term Tai Chi exercise on cardiovascular disease and its risk factors," Rehabilitation Theory and Practice in China, vol. 18, pp. 11481150, 2012.

[59] J. Emberson, P. Whincup, R. Morris, M. Walker, and S. Ebrahim, "Evaluating the impact of population and high-risk strategies for the primary prevention of cardiovascular disease," European Heart Journal, vol. 25, no. 6, pp. 484-491, 2004.

[60] Y. Zeng, T. Luo, H. Xie, M. Huang, and A. S. K. Cheng, "Health benefits of qigong or tai chi for cancer patients: a systematic review and meta-analyses," Complementary Therapies in Medicine, vol. 22, no. 1, pp. 173-186, 2014.
[61] M. E. Hackney and S. L. Wolf, "Impact of Tai Chi Chu'an practice on balance and mobility in older adults: an integrative review of 20 years of research," Journal of Geriatric Physical Therapy. In press.

[62] G. Raman, Y. Zhang, V. Minichiello, C. D’Ambrosio, and C. Wang, "Tai Chi and sleep quality in adults: a systematic review and meta-analysis," The Journal of Alternative and Complementary Medicine, vol. 20, no. 5, p. A66, 2014.

[63] M. S. Lee, M. H. Pittler, B.-C. Shin, and E. Ernst, "Tai Chi for osteoporosis: a systematic review," Osteoporosis International, vol. 19, no. 2, pp. 139-146, 2008.

[64] G. Raman, S. Mudedla, and C. Wang, "How effective is Tai Chi mind-body therapy for fibromyalgia: a systematic review and meta-analysis," The Journal of Alternative and Complementary Medicine, vol. 20, no. 5, p. A66, 2014.

[65] M. S. Lee, M. H. Pittler, and E. Ernst, "Tai chi for rheumatoid arthritis: systematic review," Rheumatology, vol. 46, no. 11, pp. 1648-1651, 2007.

[66] R. E. Taylor-Piliae and W. L. Haskell, "Tai Chi exercise and stroke rehabilitation," Topics in Stroke Rehabilitation, vol. 14, no. 4, pp. 9-22, 2007.

[67] A. Dalusung-Angosta, "The impact of Tai Chi exercise on coronary heart disease: a systematic review," Journal of the American Academy of Nurse Practitioners, vol. 23, no. 7, pp. 376381, 2011.

[68] E. H. Hwang, M. Hwang, B. C. Shin, and G. S. Lee, "Effect of Tai Chi as therapeutic exercise on blood pressure: rapid systematic review," The Journal of Alternative and Complementary Medicine, vol. 20, no. 5, article A57, 2014.

[69] L. Hartley, N. Flowers, M. S. Lee, E. Ernst, and K. Rees, "Tai chi for primary prevention of cardiovascular," The Cochrane Database of Systematic Reviews, vol. 4, Article ID CD010366, 2014. 


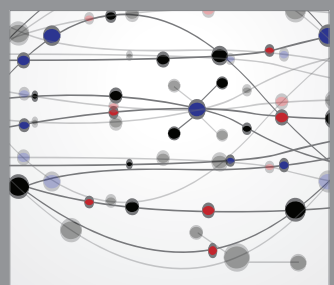

The Scientific World Journal
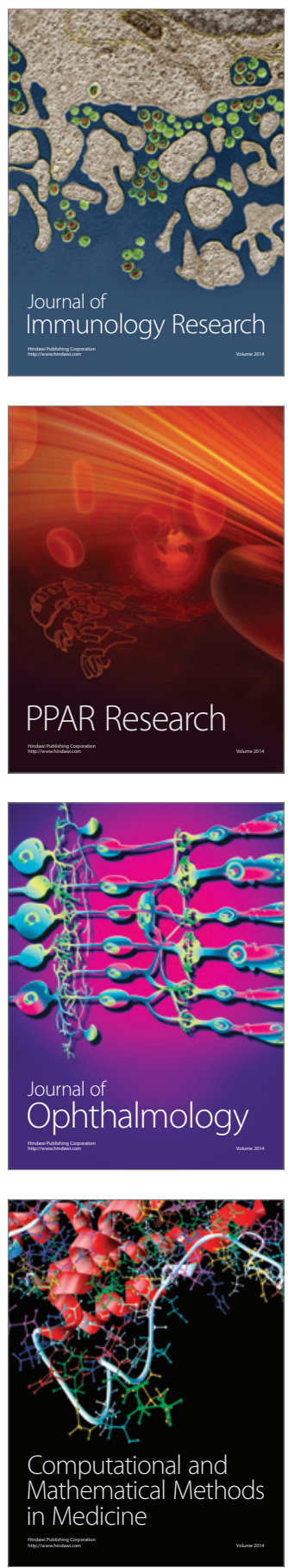

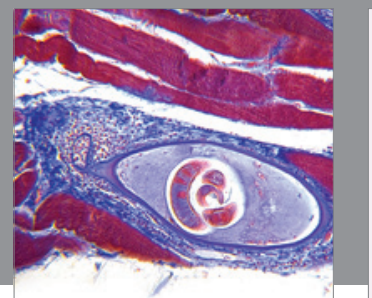

Gastroenterology

Research and Practice
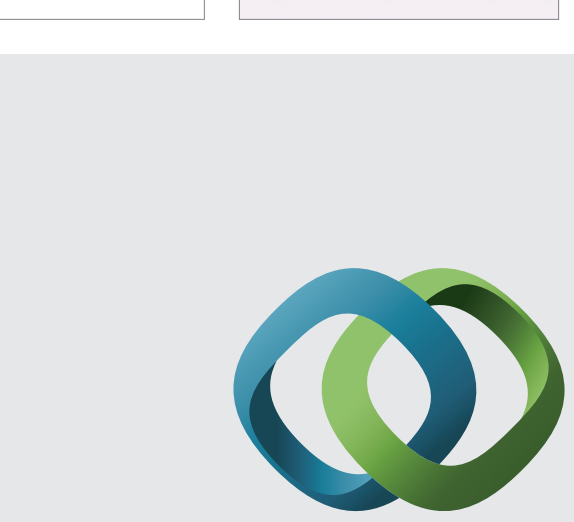

\section{Hindawi}

Submit your manuscripts at

http://www.hindawi.com
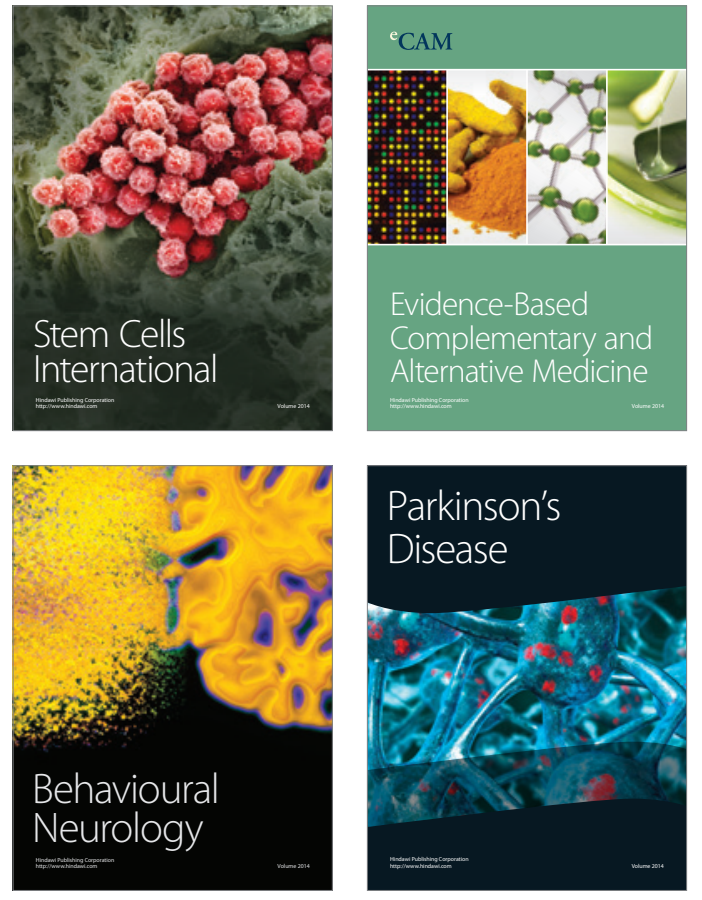
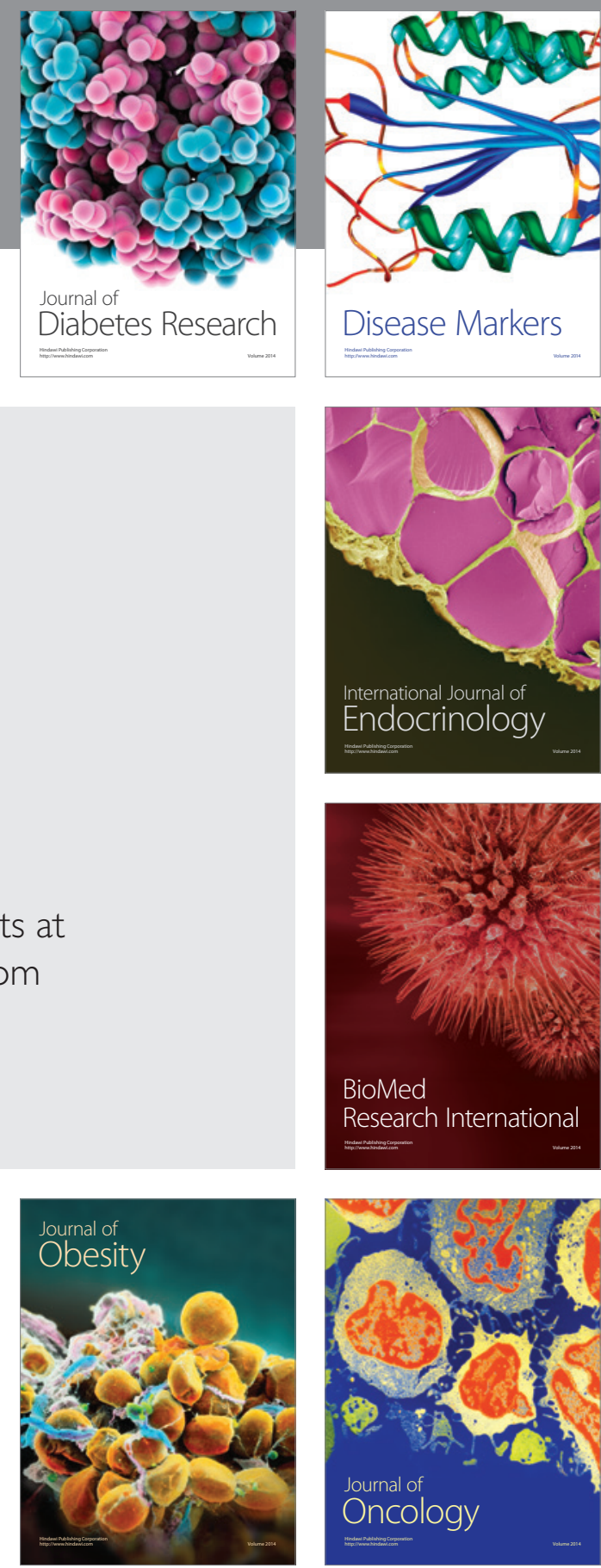

Disease Markers
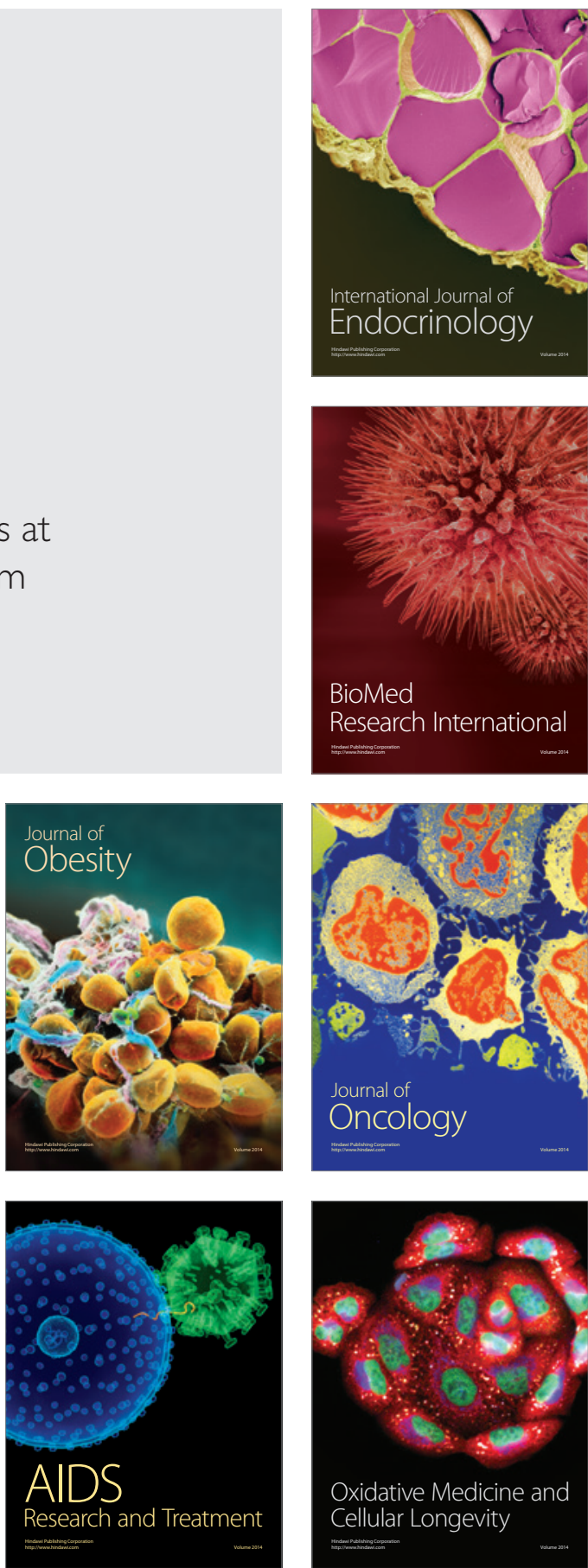